\title{
FLOWS IN FIBERS
}

\author{
JUN-ICHI TANAKA
}

Dedicated to Professor Morisuke Hasumi on his 60th birthday

\begin{abstract}
Let $H^{\infty}(\Delta)$ be the algebra of all bounded analytic functions on the open unit disc $\Delta$, and let $\mathfrak{M}\left(H^{\infty}(\Delta)\right)$ be the maximal ideal space of $H^{\infty}(\Delta)$. Using a flow, we represent a reasonable portion of a fiber in $\mathfrak{M}\left(H^{\infty}(\Delta)\right)$. This indicates a relation between the corona theorem and the individual ergodic theorem. As an application, we answer a question of Forelli [3] by showing that there exists a minimal flow on which the induced uniform algebra is not a Dirichlet algebra. The proof rests on the fact that the closure of a nonhomeomorphic part in $\mathfrak{M}\left(H^{\infty}(\Delta)\right)$ may contain a homeomorphic copy of $\mathfrak{M}\left(H^{\infty}(\Delta)\right)$. Taking suitable factors, we may construct a lot of minimal flows which are not strictly ergodic.
\end{abstract}

\section{INTRODUCTION}

If $|\alpha|=1$, then the fiber $\mathfrak{M}_{\alpha}$ of $\mathfrak{M}\left(H^{\infty}(\Delta)\right)$ over $\alpha$ is defined to be

$$
\mathfrak{M}_{\alpha}=\left\{\xi \in \mathfrak{M}\left(H^{\infty}(\Delta)\right) ; \xi(z)=\alpha\right\},
$$

where $z$ is the coordinate function. Then we have the decomposition

$$
\mathfrak{M}\left(H^{\infty}(\Delta)\right) \backslash \Delta=\bigcup_{|\alpha|=1} \mathfrak{M}_{\alpha} .
$$

Since the various fibers $\mathfrak{M}_{\alpha}$ are homeomorphic to one another, we restrict our attention to the fiber $\mathfrak{M}_{1}$ over 1 to look into the fringe $\mathfrak{M}\left(H^{\infty}(\Delta)\right) \backslash \Delta$.

Let $\Omega$ be a compact Hausdorff space on which the real line $\mathbf{R}$ acts as a topological transformation group. This means that there is a one-parameter group $\left\{U_{t}\right\}_{t \in \mathbf{R}}$ of homeomorphisms of $\Omega$ onto itself such that the map $(\omega, t) \rightarrow U_{t} \omega$ is continuous on $\Omega \times \mathbf{R}$. The pair $\left(\Omega,\left\{U_{t}\right\}_{t \in \mathbf{R}}\right)$ is referred to as a (continuous) flow. For each $\omega$ in $\Omega$ and $t$ in $\mathbf{R}$, the translate $U_{t} \omega$ of $\omega$ by $t$ is denoted by $\omega+t$. We denote by $O(\omega)$ the orbit of $\omega$, that is,

$$
O(\omega)=\{\omega+t ; t \in \mathbf{R}\} .
$$

Then an $\omega$ in $\Omega$ is said to be fixed if $O(\omega)$ equals $\{\omega\}$. A flow $\left(\Omega,\left\{U_{t}\right\}_{t \in \mathbf{R}}\right)$ is called minimal if each orbit is dense in $\Omega$, so there are no nontrivial closed invariant sets.

Received by the editors February 19, 1992 and, in revised form, November 25, 1992.

1991 Mathematics Subject Classification. Primary 46J15, 46J10; Secondary 30H05, 54H20.

Key words and phrases. Maximal ideal spaces, fibers, Gleason parts, minimal flows, Dirichlet algebras.

This research was partially supported by Grants 01540150,03640174 and 04640183 from the Japanese Ministry of Education. 
Let $H^{\infty}\left(\mathbf{R}_{+}^{2}\right)$ denote the space of all bounded analytic functions in the upper half-plane $\mathbf{R}_{+}^{2}$, and the space of their boundary-value functions is denoted by $H^{\infty}(\mathbf{R})$. They are identified each other by Fatou's theorem. Let $C(\Omega)$ be the space of all continuous complex-valued functions on $\Omega$. A function $\phi$ in $C(\Omega)$ is analytic if the function $t \rightarrow \phi(\omega+t)$ lies in $H^{\infty}(\mathbf{R})$ for each $\omega$ in $\Omega$. Let $A(\Omega)$ be the space of all analytic functions in $C(\Omega)$. Then $A(\Omega)$ is a uniformly closed subalgebra of $C(\Omega)$ containing constants. When there are no fixed points, $A(\Omega)$ separates the points on $\Omega$, and $\Omega$ is the Choquet boundary of $A(\Omega)$ [13, Theorem I]. Throughout the paper, we shall always assume that $\left(\Omega,\left\{U_{t}\right\}_{t \in \mathbf{R}}\right)$ is an arbitrary flow without fixed points. Then $A(\Omega)$ becomes a uniform algebra on $\Omega$, which is called the induced uniform algebra on $\left(\Omega,\left\{U_{t}\right\}_{t \in \mathbf{R}}\right)$. Let $\mathfrak{M}(A(\Omega))$ be the maximal ideal space of $A(\Omega)$. Although the structure is intractable, it has been investigated with the aid of ergodic theory (see, for instance, [12, 16 and 15]).

Let $\mathbf{A}$ be a uniform algebra on a compact Hausdorff space $\mathbf{Y}$. Then $\mathbf{A}$ is called a Dirichlet algebra on $\mathbf{Y}$ if the real part $\operatorname{Re} \mathbf{A}$ of $\mathbf{A}$ is uniformly dense in the space $C_{\mathbf{R}}(\mathbf{Y})$ of all real-valued functions in $C(\mathbf{Y})$. We say that $\mathbf{A}$ is a logmodular algebra on $\mathbf{Y}$ if

$$
\log \left|\mathbf{A}^{-1}\right|=\left\{\log |f| ; f \in \mathbf{A}^{-1}\right\}
$$

is uniformly dense in $C_{\mathbf{R}}(\mathbf{Y})$, where $\mathbf{A}^{-1}$ denotes the set of all invertible elements of $\mathbf{A}$.

The purpose of this paper is to show how flows can be applied usefully to study the structure of $\mathfrak{M}\left(H^{\infty}(\Delta)\right)$. We first represent a portion of the fiber $\mathfrak{M}_{1}$ as a subset of the maximal ideal space of the induced uniform algebra on a flow. Then we point out that there appears a relation between the corona theorem and the individual ergodic theorem. Together with the characterization of analytic structure in $\mathfrak{M}\left(H^{\infty}(\Delta)\right)$, due to Hoffman, we second construct a minimal flow on which the induced uniform algebra is not a Dirichlet algebra. This provides a negative answer to a question posed by Forelli [3]:

When $\left(\Omega,\left\{U_{t}\right\}_{t \in \mathbf{R}}\right)$ is minimal, is $A(\Omega)$ a Dirichlet algebra on $\Omega$ ?

In the next section, we establish the notation and present some lemmas. Our representation of the fiber $\mathfrak{M}_{1}$ over 1 is given in $\S 3$ together with some related facts. After preparing some lemmas, we show in $\S 4$ the existence of a desired minimal flow. We close with some remarks in $\S 5$.

The author would like to thank Professor Keiji Izuchi for several informative conversations. He is also grateful to the referee for his valuable suggestions which improved the first version of this paper.

\section{Preliminaries}

We begin by collecting some notation and facts about a flow $\left(\Omega,\left\{U_{t}\right\}_{t \in \mathbf{R}}\right)$ which we shall use throughout. Let $M(\Omega)$ be the space of all bounded complex Borel regular measures on $\Omega$. A measure in $M(\Omega)$ is said to be quasi-invariant if every translate of each null set is also a null set. A quasi-invariant measure is said to be ergodic if every invariant subset of $\Omega$ is either negligible or has negligible complement. Let $\mu$ be a positive quasi-invariant measure. A function $\phi$ in $L^{\infty}(\mu)$ is said to be analytic if the function $t \rightarrow \phi(\omega+t)$ lies in $H^{\infty}(\mathbf{R})$ for $\mu$-a.e. $\omega$ in $\Omega$. 
Let us consider the representing measures for the induced uniform algebra $A(\Omega)$ on $\left(\Omega,\left\{U_{t}\right\}_{t \in \mathbf{R}}\right)$. The Poisson kernel $P_{z}(t)$ for $\mathbf{R}_{+}^{2}$ is defined by $P_{z}(t)=$ $s / \pi\left(s^{2}+(t-u)^{2}\right)$, where $z=u+i s$. For each $s>0$ and for a point $\omega+u$ in the orbit $O(\omega)$ of $\omega$, we define a homomorphism of $A(\Omega)$ by

$$
\xi_{z}(\phi)=\phi * P_{i s}(\omega+u)=\int_{-\infty}^{\infty} \phi(\omega+t) P_{z}(t) d t
$$

Then the product $O(\omega) \times(0, \infty)$ may be regarded as a subset of $\mathfrak{M}(A(\Omega))$ by (2.1). Since the representing measure for each point in $O(\omega) \times(0, \infty)$ is absolutely continuous to another one, $O(\omega) \times(0, \infty)$ makes a nontrivial Gleason part. On the other hand, there are representing measures of a different kind. Indeed, the Markov-Kakutani theorem shows that there is at least one invariant probability measure on $\Omega$. Together with the Krein-Milman theorem, this assures the existence of an invariant, ergodic, probability measure $m$ on $\Omega$. Then $m$ is a representing measure for $A(\Omega)$ [11, Theorem I]. It is known that every representing measure for $A(\Omega)$ is quasi-invariant if it is not a point mass [11, Theorem III].

Let $\beta \mathbf{Z}$ be the Stone-Čech compactification of the group $\mathbf{Z}$ of integers, and let $S_{0}$ be the shift operator on $\mathbf{Z}$, which is defined by $S_{0} n=n+1$ for each $n$ in $\mathbf{Z}$. It then follows from the Banach-Stone theorem [1, Chapter $\mathbf{V}, 8.8$ ] that $S_{0}$ extends to a homeomorphism $\mathbf{S}$ of $\beta \mathbf{Z}$ onto itself. We denote by $\mathbf{X}$ the quotient space obtained from $\beta \mathbf{Z} \times[0,1]$ by identifying $(y, 1)$ with $(\mathbf{S} y, 0)$ for each $y$ in $\beta \mathbf{Z}$. Then $\mathbf{X}$ is a compact Hausdorff space. Let us define a flow $\left(\mathbf{X},\left\{\mathbf{S}_{t}\right\}_{t \in \mathbf{R}}\right)$ by

$$
\mathbf{S}_{t}(y, s)=\left(\mathbf{S}^{[t+s]} y, t+s-[t+s]\right),
$$

where $[t]$ denotes the largest integer not exceeding $t$. We notice that the flow is far from a minimal one, since there exist closed invariant sets in profusion. Then there are many invariant, ergodic, probability measures on $M(\mathbf{X})$. We not only see that there are no fixed points in $\mathbf{X}$ but also that there are no periodic orbits in $\mathbf{X}$. Then $A(\mathbf{X})$ is a uniform algebra on $\mathbf{X}$.

Let $X_{0}$ be the screw with pitch 1 and radius $1 / 2 \pi$, that is, the quotient space obtained from $\mathbf{Z} \times[0,1]$ by identifying each $(n, 1)$ with $(n+1,0)$. Since $\mathbf{Z}$ is dense $\beta \mathbf{Z}, X_{0}$ is also dense in $\mathbf{X}$. The map $t \rightarrow([t], t-[t])$ is a homeomorphism of $\mathbf{R}$ onto the subspace $X_{0}$ of $\mathbf{X}$. In what follows, we shall sometimes identify $X_{0}$ and $X_{0} \times(0, \infty)$ with $\mathbf{R}$ and $\mathbf{R}_{+}^{2}$, respectively. Then $\mathbf{R}_{+}^{2}$ may be considered as a subset of $\mathfrak{M}(A(\mathbf{X}))$ by $(2.1)$. Let $C_{b}(\mathbf{R})$ be the space of all bounded continuous functions on $\mathbf{R}$. Then each function in $C(\mathbf{X})$ determines a function in $C_{b}(\mathbf{R})$ by restricting it to $X_{0}$.

Every function $\phi$ on $\mathbf{X}$ has the automorphic extention $\phi^{\#}$ to $\beta \mathbf{Z} \times \mathbf{R}$ by

$$
\phi^{\#}(y, t)=\phi\left(\mathbf{S}^{[t]} y, t-[t]\right) .
$$

A function $f$ on $\beta \mathbf{Z} \times \mathbf{R}$ is the automorphic extension of a function on $\mathbf{X}$ if and only if $f(y, t)=f(\mathbf{S} y, t-1)$ on $\beta \mathbf{Z} \times \mathbf{R}$.

Lemma 2.1. Let $\psi_{0}$ be a function in $C_{b}(\mathbf{R})$. Then $\psi_{0}$ extends to a function $\psi$ in $C(\mathbf{X})$ if and only if $\psi_{0}$ is uniformly continuous on $\mathbf{R}$.

Proof. Suppose that $\psi_{0}$ is uniformly continuous on $\mathbf{R}$. For each $s$ in $[0,1)$, the sequence $\psi_{0}(n, s)=\psi_{0}(n+s), n \in \mathbf{Z}$, lies in $l^{\infty}(\mathbf{Z})$. Then $\psi_{0}(n, s)$ 
extends to $\psi(y, s)$ in $C(\beta \mathbf{Z})$. Since $\mathbf{X}$ is identified with $\beta \mathbf{Z} \times[0,1)$, we may regard $\psi(y, s)$ as a function on $\mathbf{X}$. Let $\psi^{\sharp}(y, t)$ be the automorphic extension of $\psi(y, s)$ to $\beta \mathbf{Z} \times \mathbf{R}$. It follows from the uniform continuity of $\psi_{0}$ that the family $\left\{\psi^{\#}(y, t) ; y \in \beta \mathbf{Z}\right\}$ of the functions of $t$ is equicontinuous on $\mathbf{R}$. This implies that $\psi^{\sharp}$ is continuous on $\beta \mathbf{Z} \times \mathbf{R}$. Thus $\psi$ lies in $C(\mathbf{X})$.

The converse follows from a general fact. Indeed, it is an immediate consequence of the continuity of the map $(x, t) \rightarrow x+t$ on $\mathbf{X} \times \mathbf{R}$.

Let $C_{b u}(\mathbf{R})$ be the space of bounded uniformly continuous functions on $\mathbf{R}$. Then $C_{b u}(\mathbf{R})$ is a Banach algebra with the supremum norm, which is not separable. Lemma 2.1 shows that $\mathbf{X}$ is the maximal ideal space of $C_{b u}(\mathbf{R})$ and that the flow $\left\{\mathbf{S}_{t}\right\}_{t \in \mathbf{R}}$ on $\mathbf{X}$ is that which is induced by the natural translations of the functions in $C_{b u}(\mathbf{R})$. Such observation may provide an approach to almost periodic functions.

Lemma 2.2. Let $A(\mathbf{X})$ be the induced uniform algebra on $\left(\mathbf{X},\left\{\mathbf{S}_{t}\right\}_{t \in \mathbf{R}}\right)$, and let $\phi_{0}$ be a function in $H^{\infty}(\mathbf{R})$. If $r>0$, then the convolution $\phi_{0} * P_{i r}$, in the usual sense, extends to a function $\phi_{r}(x)$ in $A(\mathbf{X})$. Furthermore, the function $(x, r) \rightarrow \phi_{r}(x)$ is continuous on $\mathbf{X} \times(0, \infty)$.

Proof. Since

$$
\left|\phi_{0} * P_{i r}(t-s)-\phi_{0} * P_{i r}(t)\right| \leq\left\|\phi_{0}\right\|_{\infty} \cdot\left\|P_{s+i r}-P_{i r}\right\|_{1},
$$

$t \rightarrow \phi_{0} * P_{i r}(t)$ is uniformly continuous on $\mathbf{R}$. By Lemma 2.1, $\phi_{0} * P_{i r}$ extends to a $\phi_{r}(x)$ in $C(\mathbf{X})$. Let us consider the automorphic extension $\phi_{r}^{\#}(y, t)$ of $\phi_{r}$ to $\beta \mathbf{Z} \times \mathbf{R}$. Then $t \rightarrow \phi_{r}^{\#}(y, t)$ extends to $\mathbf{R}_{+}^{2}$ by its Poisson integral:

$$
\phi_{r}^{\#}(y, z)=\phi_{r}^{\#}(y, t+i s)=\phi_{r}^{\#} * P_{i s}(y, t) .
$$

Since $\phi_{0} * P_{i r}$ lies in $H^{\infty}(\mathbf{R}), z \rightarrow \phi_{r}^{\#}(n, z)$ is bounded analytic on $\mathbf{R}_{+}^{2}$ for each $n$ in $\mathbf{Z}$. Then, since $\mathbf{Z}$ is dense in $\beta \mathbf{Z}, z \rightarrow \phi_{r}^{\#}(y, z)$ lies also in $H^{\infty}\left(\mathbf{R}_{+}^{2}\right)$ for each $y$ in $\beta \mathbf{Z}$. This shows that $t \rightarrow \phi_{r}(x+t)$ lies in $H^{\infty}(\mathbf{R})$. Thus $\phi_{r}$ is a function in $A(\mathbf{X})$.

Since $\phi_{0} * P_{i r} * P_{i s}=\phi_{0} * P_{i(r+s)}$ for $s>0$, we see that $\phi_{r}^{\#}(y, t+i s)=\phi_{r+s}^{\#}(y, t)$ by (2.4). Then the family $\left\{\phi_{r}^{*}(y, t+i s) ; y \in \beta \mathbf{Z}\right\}$ of functions of $t+i s$ is equicontinuous on $\mathbf{R}_{+}^{2}$. This implies that $(x, s) \rightarrow \phi_{r+s}(x)$ is continuous on $\mathbf{X} \times(0, \infty)$ for each $r>0$. From this, the continuity of $(x, r) \rightarrow \phi_{r}(x)$ follows.

Proposition 2.3. Let $A(\mathbf{X})$ be the induced uniform algebra on $\left(\mathbf{X},\left\{\mathbf{S}_{t}\right\}_{t \in \mathbf{R}}\right)$. Then $A(\mathbf{X})$ is a logmodular algebra on $\mathbf{X}$ which is not a Dirichlet algebra.

Proof. Suppose $\phi$ lies in $C_{\mathbf{R}}(\mathbf{X})$. For a given $\varepsilon>0$, we choose an $r>0$ such that $\left\|\phi-\phi * P_{i r}\right\|_{\infty}<\varepsilon$ by [2, Lemma 1]. On the other hand, the restriction $\phi_{0}$ of $\phi$ to $X_{0}$ lies in $C_{b}(\mathbf{R})$. Let $H \phi_{0}$ be the Hilbert transform of $\phi_{0}$ defined by

$$
H \phi_{0}(t)=\lim _{\varepsilon \rightarrow+0} \frac{1}{\pi} \int_{|t-s|>\varepsilon} \phi_{0}(s)\left[\frac{1}{t-s}+\frac{s}{1+s^{2}}\right] d s .
$$

Then $\psi_{0}=\exp \left(\phi_{0}+i H \phi_{0}\right)$ is an outer function in $H^{\infty}(\mathbf{R})$, which is invertible in $H^{\infty}(\mathbf{R})$. Observe that $\psi_{0}^{-1} * P_{i r}(t)$ is the inverse of $\psi_{0} * P_{i r}(t)$ in $H^{\infty}(\mathbf{R})$. Since $\log \left|\psi_{0}\right|=\phi_{0}$, we see that

$$
\log \left|\psi_{0} * P_{i r}(t)\right|=\phi_{0} * P_{i r}(t) .
$$


It follows from Lemma 2.2 that $\psi_{0} * P_{i r}(t)$ and $\psi_{0}^{-1} * P_{i r}(t)$ extend to $\theta$ and $\theta^{-1}$ in $A(\mathbf{X})$. We see easily that $\log |\theta|=\phi * P_{i r}$ by (2.6). So $A(\mathbf{X})$ is a logmodular algebra on $\mathbf{X}$.

We now show that $A(\mathbf{X})$ is not a Dirichlet algebra. Let $u_{0}(t)=\frac{2}{\pi} \tan ^{-1}(t)$. Since $u_{0}$ is uniformly continuous on $\mathbf{R}, u_{0}$ extends to a function $u$ in $C_{\mathbf{R}}(\mathbf{X})$. However, we observe by $(2.5)$ that

$$
\operatorname{dist}\left(u_{0}, \operatorname{Re} H^{\infty}(\mathbf{R})\right)=\inf \left\{\left\|u_{0}-\operatorname{Re} \psi_{0}\right\|_{\infty} ; \psi_{0} \in H^{\infty}(\mathbf{R})\right\}=1
$$

(see [6, Chapter IV, Example 1.5 and Chapter V, Exercise 15]). Thus $u$ cannot belong to the closure of $\operatorname{Re} A(\mathbf{X})$ in $C_{\mathbf{R}}(\mathbf{X})$.

Let $\nu_{0}$ be the measure on $\mathbf{Z}$ defined by $\nu_{0}(\{n\})=1 /\left(n^{2}+1\right)$, and let $m_{I}$ be the restriction of Lebesgue measure to $I=[0,1]$. By identifying $\mathbf{R}$ with $X_{0}$, $d t /\left(1+t^{2}\right)$ and $\nu_{0} \times m_{I}$ are mutually absolutely continuous. Since $l^{\infty}(\mathbf{Z})=$ $L^{\infty}\left(\nu_{0}\right), L^{\infty}\left(\nu_{0}\right)$ is isometrically isomorphic to $C(\beta \mathbf{Z})$ and $\nu_{0}$ extends to a normal measure $\nu$ in $M(\beta \mathbf{Z})$ (see [5, Chapter I, $\S 9]$ ). We then see that $\nu$ is quasi-invariant with respect to $\mathbf{S}$, to be exact, $\nu$-null sets are preserved under the translation by $\mathbf{S}$. Therefore the measure $\nu \times m_{I}$ in $M(\mathbf{X})$ is a quasiinvariant measure on $\left(\mathbf{X},\left\{\mathbf{S}_{t}\right\}_{t \in \mathbf{R}}\right)$ by the definition (2.2).

Let $H^{\infty}(\mathbf{X})$ be the space of all analytic functions in $L^{\infty}\left(\nu \times m_{I}\right)$. Since $\nu \times$ $m_{I}$ is concentrated on $X_{0}$, that is, $\nu \times m_{I}\left(\mathbf{X} \backslash X_{0}\right)=0, H^{\infty}(\mathbf{R})$ is isometrically isomorphic to $H^{\infty}(\mathbf{X})$ by

$$
\Phi_{0} \phi_{0}(n, s)=\phi_{0}(n+s), \quad \phi_{0} \in H^{\infty}(\mathbf{R}),
$$

for $(n, s)$ in $X_{0}$. Since the restriction of each function in $H^{\infty}(\mathbf{X})$ to $X_{0}$ determines a function in $H^{\infty}(\mathbf{R}), \Phi_{0}$ maps $H^{\infty}(\mathbf{R})$ onto $H^{\infty}(\mathbf{X})$. Let $\mathfrak{M}\left(H^{\infty}(\mathbf{R})\right)$ and $\mathfrak{M}\left(H^{\infty}(\mathbf{X})\right)$ be the maximal ideal spaces of $H^{\infty}(\mathbf{R})$ and $H^{\infty}(\mathbf{X})$. Then the adjoint $\Phi_{0}^{*}$ of $\Phi_{0}$ maps homeomorphically $\mathfrak{M}\left(H^{\infty}(\mathbf{X})\right)$ onto $\mathfrak{M}\left(H^{\infty}(\mathbf{R})\right)$. We shall modify $\Phi_{0}$ in a little while.

The important feature of $H^{\infty}(\mathbf{X})$ is that each element has a useful Borel version.

Lemma 2.4. Let $\phi$ be a function in $H^{\infty}(\mathbf{X})$. Then there is a Borel function $\tilde{\phi}$ on $\mathbf{X}$ with the following properties:

(i) The equation $\phi(x)=\tilde{\phi}(x)$ holds for $\nu \times m_{I}$-a.e. $x$ in $\mathbf{X}$.

(ii) The function $x \rightarrow \tilde{\phi} * P_{\text {ir }}(x)$ lies in $A(\mathbf{X})$ for each $r>0$. Furthermore, the function $(x, r) \rightarrow \tilde{\phi} * P_{i r}(x)$ is continuous on $\mathbf{X} \times(0, \infty)$.

(iii) Let $\mu$ be a positive quasi-invariant measure on $\mathbf{X}$. Then we obtain

$$
\lim _{r \rightarrow+0} \int_{\mathbf{X}} \tilde{\phi} * P_{i r}(x) d \mu(x)=\int_{\mathbf{X}} \tilde{\phi}(x) d \mu(x) .
$$

Proof. We define the Borel function $\tilde{\phi}$ for a given $\phi$ in $H^{\infty}(\mathbf{X})$. Let $\phi_{0}$ be the restriction of $\phi$ to $X_{0}$. Then $\phi_{0}$ is regarded as a function in $H^{\infty}(\mathbf{R})$. By Lemma $2.2, \phi_{0} * P_{i r}$ extends to a function $\phi_{r}(x)$ in $A(\mathbf{X})$, and $(x, r) \rightarrow \phi_{r}(x)$ is continuous on $\mathbf{X} \times(0, \infty)$. We set

$$
f(y, z)=f(y, t+i r)=\phi_{r}^{\#}(y, t),
$$

where $\phi_{r}^{\#}(y, t)$ is the automorphic extension of $\phi_{r}$ to $\beta \mathbf{Z} \times \mathbf{R}$. So $f$ is continuous on $\beta \mathbf{Z} \times \mathbf{R}_{+}^{2}$, and $z \rightarrow f(y, z)$ lies in $H^{\infty}\left(\mathbf{R}_{+}^{2}\right)$ for each $y$ in $\beta \mathbf{Z}$. 
Let $\left\{r_{n}\right\}$ be a decreasing sequence that converges to 0 . We then define the boundary-value function $f(y, t)$ by

$$
f(y, t)=\limsup _{n \rightarrow \infty} \operatorname{Re} f\left(y, t+i r_{n}\right)+i \limsup _{n \rightarrow \infty} \operatorname{Im} f\left(y, t+i r_{n}\right) .
$$

Since $(y, t) \rightarrow f\left(y, t+i r_{n}\right)$ is continuous on $\beta \mathbf{Z} \times \mathbf{R}, f(y, t)$ is a Borel function on $\beta \mathbf{Z} \times \mathbf{R}$. On the other hand, it follows from Fatou's theorem that, for each $y$ in $\beta \mathbf{Z}$,

$$
f(y, t)=\lim _{r \rightarrow+0} f(y, t+i r)
$$

for $d t$-a.e. $t$ in $\mathbf{R}$. Since $f(y, t)=f(\mathbf{S} y, t-1)$ by (2.9), there is a Borel function $\tilde{\phi}$ on $\mathbf{X}$ whose automorphic extension is $f(y, t)$.

Since (2.10) shows that if $n$ lies in $\mathbf{Z}$, then

$$
\lim _{r \rightarrow+0} \phi_{0} * P_{i r}(n+t)=\phi_{0}(n+t)=f(n, t)
$$

for $d t$-a.e. $t$, we see that $\phi^{\#}(y, t)=f(y, t)$ for $d \nu \times d t$-a.e. $(y, t)$. Thus (i) holds. We see easily that $\tilde{\phi} * P_{i r}(x)=\phi_{r}(x)$ on $\mathbf{X}$. Then (ii) follows from Lemma 2.2. To show (iii), we define the measure $\mu_{1}$ in $M(\beta \mathbf{Z})$ by $\mu_{1}(E)=\mu(E \times[0,1])$. Then $\mu_{1}$ is quasi-invariant with respect to $\mathbf{S}$. It is easy to see that $\mu_{1} \times m_{I}$ is a quasi-invariant measure on $\mathbf{X}$ which is mutually absolutely continuous with respect to $\mu$. Identifying $\mathbf{X}$ with $\beta \mathbf{Z} \times[0,1)$,

$$
\lim _{r \rightarrow+0} \tilde{\phi} * P_{i r}(y, s)=\tilde{\phi}(y, s)
$$

holds for $m_{I}$-a.e. $s$ in $[0,1)$ and for each $y$ in $\beta \mathbf{Z}$. Thus (2.8) follows from Fubini's theorem and the bounded convergence theorem.

Let $\Phi_{0}$ be the isometric isomorphism of $H^{\infty}(\mathbf{R})$ onto $H^{\infty}(\mathbf{X})$ by (2.7). For a given $\phi_{0}$ in $H^{\infty}(\mathbf{R})$, we set $\phi=\Phi_{0}\left(\phi_{0}\right)$. Strictly speaking, $\phi$ is an equivalence class of functions modulo null functions. Then we assign the Borel function $\tilde{\phi}$ by Lemma 2.4 to $\Phi_{0}\left(\phi_{0}\right)$ :

$$
\Phi_{0}\left(\phi_{0}\right)=\tilde{\phi}, \quad \phi_{0} \in H^{\infty}(\mathbf{R}) .
$$

From now on, $\Phi_{0}$ will denote this modified isomorphism.

By virtue of this specialization, certain homomorphisms of $A(\mathbf{X})$ become the ones of $H^{\infty}(\mathbf{R})$. Since $H^{\infty}(\mathbf{R})$ and $H^{\infty}(\mathbf{X})$ are isometrically isomorphic via $\Phi_{0}$, it is a consequence of the following lemma, in which the uniqueness of extension depends on the corona theorem.

Lemma 2.5. Suppose that $\xi_{0}$ lies in $\mathfrak{M}(A(\mathbf{X})) \backslash \mathbf{X}$. Then $\xi_{0}$ extends uniquely to an element $\xi$ of $\mathfrak{M}\left(H^{\infty}(\mathbf{X})\right)$.

Proof. Let $\mu$ be the representing measure for $\xi_{0}$. Since $\mu$ is not a point mass, $\mu$ is quasi-invariant on $\mathbf{X}$. Using the Borel version by Lemma 2.4, we define

$$
\xi(\phi)=\int_{\mathbf{X}} \tilde{\phi} d \mu, \quad \phi \in H^{\infty}(\mathbf{X}) .
$$

Since $(\phi \psi)^{\sim}=\tilde{\phi} \tilde{\psi}$ if $\phi$ and $\psi$ lie in $H^{\infty}(\mathbf{X})$, it follows from (2.8) that $\xi$ is a homomorphism of $H^{\infty}(\mathbf{X})$ which is an extension of $\xi_{0}$.

The uniqueness of $\xi$ follows from the corona theorem. Since $H^{\infty}(\mathbf{R})$ is isometrically isomorphic to $H^{\infty}(\mathbf{X})$, the corona theorem asserts that $X_{0} \times(0, \infty)$ 
is dense in $\mathfrak{M}\left(H^{\infty}(\mathbf{X})\right)$ by (2.1). Observe that $t \rightarrow e^{i t}$ is uniformly continuous on $\mathbf{R}$. Then Lemma 2.1 implies that $e^{i t}$ extends to a function $s_{1}(x)$ in $A(\mathbf{X})$ such that $\left|s_{1}(x)\right|=1$ on $\mathbf{X}$. Since $t \rightarrow s_{1}(x+t)$ is periodic, we see that $s_{1}$ is not constant as a function in $L^{1}(\mu)$. Then there is an $r$ with $0<r<1$ such that

$$
\left|\xi_{0}\left(s_{1}\right)\right|=\left|\int_{\mathbf{X}} s_{1} d \mu\right| \leq r .
$$

Suppose $\xi^{\prime}$ in $\mathfrak{M}\left(H^{\infty}(\mathbf{X})\right)$ satisfies that $\xi^{\prime}=\xi_{0}$ on $A(\mathbf{X})$. Since $A(\mathbf{X})$ is a logmodular algebra on $\mathbf{X}$, the representing measure $\mu$ for $\xi_{0}$ is unique. By the corona theorem and (2.13), there is a net $\left\{\xi_{\alpha}\right\}$ in $X_{0} \times[-\log r, \infty)$ such that $\left\{\xi_{\alpha}\right\}$ converges to $\xi^{\prime}$ in $\mathfrak{M}\left(H^{\infty}(\mathbf{X})\right)$. By setting $\xi_{\alpha}=\left(x_{\alpha}, t_{\alpha}\right),(2.1)$ and (ii) of Lemma 2.4 show that

$$
\int_{-\infty}^{\infty} \tilde{\phi} * P_{i u}\left(x_{\alpha}+t\right) P_{i t_{\alpha}}(t) d t \rightarrow \int_{\mathbf{X}} \tilde{\phi} * P_{i u} d \mu
$$

for each $\phi$ in $H^{\infty}(\mathbf{X})$ and for $u>0$. Furthermore, we see that if $\left\{t_{\alpha}\right\}$ is bounded, then $\mu$ is the representing measure for $\xi_{0}=(x, t)$ in $\mathbf{X} \times[-\log r, \infty)$. When $\left\{t_{\alpha}\right\}$ diverges, $\mu$ is an invariant measure on $\mathbf{X}$. In both cases, we obtain easily that

$$
\int_{-\infty}^{\infty} \tilde{\phi}\left(x_{\alpha}+t\right) P_{i t_{\alpha}}(t) d t \rightarrow \int_{\mathbf{X}} \tilde{\phi} d \mu
$$

by the condition $t_{\alpha} \geq-\log r$. This implies that $\left\{\xi_{\alpha}\right\}$ also converges to $\xi$ in $\mathfrak{M}\left(H^{\infty}(\mathbf{X})\right)$. Hence $\xi^{\prime}=\xi$, so the extension of $\xi_{0}$ is unique.

\section{IN RELATION TO FIBERS}

In this section, we represent a portion of the fiber $\mathfrak{M}_{1}$ of $\mathfrak{M}\left(H^{\infty}(\Delta)\right)$ over 1 as a subset of the maximal ideal space $\mathfrak{M}(A(\mathbf{X}))$ of $A(\mathbf{X})$. This enable us to make clearer the structure of fibers and to give information about Gleason parts in them.

Let $\mathbf{A}$ be a uniform algebra with the maximal ideal space $\mathfrak{M}(\mathbf{A})$. We usually identify each function in $\mathbf{A}$ with its Gelfand transform on $\mathfrak{M}(\mathbf{A})$. A continuous map $F$ of $\Delta$ into $\mathfrak{M}(\mathbf{A})$ is analytic if $f \circ F$ is analytic on $\Delta$ when $f$ lies in $\mathbf{A}$. A one-to-one analytic map is said to be an analytic disc. With an analytic disc, we do not distinguish between the map $F$ and its image $F(\Delta)$. Let $\mathfrak{M}(A)^{\Delta}$ be the set of all maps of $\Delta$ into $\mathfrak{M}(\mathbf{A})$. Then $\mathfrak{M}(\mathbf{A})^{\Delta}$ is a compact Hausdorff space in the product topology. If a net $\left\{F_{\beta}\right\}$ of analytic maps converges to $F$ in $\mathfrak{M}(\mathbf{A})^{\Delta}$, then $F$ is also analytic.

Observe that the function

$$
w(z)=i \frac{1+z}{1-z}, \quad z \in \Delta,
$$

maps conformally $\Delta$ onto $\mathbf{R}_{+}^{2}$. Then $w$ maps $\{-1,0,1\}$ to $\{0, i, \infty\}$. We next define the isometric isomorphism of $H^{\infty}(\Delta)$ onto $H^{\infty}\left(\mathbf{R}_{+}^{2}\right)$ by

$$
\Phi_{1}(f)=f \circ w^{-1}, \quad f \in H^{\infty}(\Delta) .
$$

To investigate the fiber $\mathfrak{M}_{1}$, we look into the behavior of functions in $H^{\infty}\left(\mathbf{R}_{+}^{2}\right)$ around at infinity. 
Let $s(z)$ be the singular function in $H^{\infty}(\Delta)$ defined by

$$
s(z)=\exp i w(z), \quad z \in \Delta .
$$

Then $s(z)$ has a singularity at $z=1$. Identifying $s$ with its Gelfand transform on $\mathfrak{M}\left(H^{\infty}(\Delta)\right)$, we set

$$
H=\left\{\xi \in \mathfrak{M}\left(H^{\infty}(\Delta)\right) ;|s(\xi)|<1\right\} .
$$

Then $H$ is an open set in $\mathfrak{M}\left(H^{\infty}(\Delta)\right)$ that contains $\Delta$. Observe that $H \backslash \Delta$ is contained in $\mathfrak{M}_{1}$. More precisely, $\xi$ in $\mathfrak{M}_{1}$ lies in $H \backslash \Delta$ if and only if $\xi$ lies in the closure of an open disc inside $\Delta$ which is tangent to the unit circle at 1 , that is, $\xi$ is orocycular or nontangential (see $[9, \S 6]$ ).

By using the flow $\left(\mathbf{X},\left\{\mathbf{S}_{t}\right\}_{t \in \mathbf{R}}\right)$ in $\S 2$, the open set $\mathfrak{M}_{1} \cap H$ in $\mathfrak{M}_{1}$ can be analyzed rather completely. Recall that we identify $X_{0} \times(0, \infty)$ with $\mathbf{R}_{+}^{2}$.

Theorem 3.1. Let $H$ be the open set in $\mathfrak{M}\left(H^{\infty}(\Delta)\right)$ defined by (3.4). Then there is a homeomorphism $\pi$ of $H$ onto $\mathfrak{M}(A(\mathbf{X})) \backslash \mathbf{X}$ with the following properties:

(i) Let $\pi_{1}$ denote the restriction of $\pi$ to $\mathfrak{M}_{1} \cap H$. Then $\pi_{1}$ maps homeomorphically $\mathfrak{M}_{1} \cap H$ onto $\mathfrak{M}(A(\mathbf{X})) \backslash\left(\mathbf{X} \cup \mathbf{R}_{+}^{2}\right)$.

(ii) The homeomorphism $\pi$ preserves Gleason metrics. Furthermore, a continuous map $F$ of $\Delta$ into $H$ is analytic if and only if so is the map $\pi \circ F$ of $\Delta$ into $\mathfrak{M}(A(\mathbf{X})) \backslash \mathbf{X}$.

Proof. Let $\Phi_{0}$ and $\Phi_{1}$ be the isometric isomorphisms by (2.11) and (3.2), respectively. Setting $\Phi=\Phi_{0} \circ \Phi_{1}$, we obtain an isometric isomorphism of $H^{\infty}(\Delta)$ onto $H^{\infty}(\mathbf{X})$ (see Figure 1). Let $\Phi^{*}$ denote the adjoint of $\Phi$. Then $\Phi^{*}$ maps homeomorphically $\mathfrak{M}\left(H^{\infty}(\mathbf{X})\right)$ onto $\mathfrak{M}\left(H^{\infty}(\Delta)\right)$. We see also that $\Phi^{*}$ is an isometry of $H^{\infty}(\mathbf{X})^{*}$ onto $H^{\infty}(\Delta)^{*}$, where $H^{\infty}(\mathbf{X})^{*}$ and $H^{\infty}(\Delta)^{*}$ denote the dual spaces of $H^{\infty}(\mathbf{X})$ and $H^{\infty}(\Delta)$. So $\Phi^{*}$ maps each Gleason part of $\mathfrak{M}\left(H^{\infty}(\mathbf{X})\right)$ to the one of $\mathfrak{M}\left(H^{\infty}(\Delta)\right)$.

As in the proof of Lemma 2.5, $s_{1}(x)$ denotes the extension of $t \rightarrow e^{i t}$ to a function in $A(\mathbf{X})$. We then set

$$
H_{1}=\left\{\xi \in \mathfrak{M}\left(H^{\infty}(\mathbf{X})\right) ;\left|s_{1}(\xi)\right|<1\right\} .
$$

Then $H_{1}$ is an open set in $\mathfrak{M}\left(H^{\infty}(\mathbf{X})\right)$. Since $\left|s_{1}(\xi)\right|=1$ on $\mathfrak{M}\left(H^{\infty}(\mathbf{X})\right) \backslash H_{1}$, $H_{1}$ is a union of Gleason parts. Since $\Phi_{1} s(t)=e^{i t}$ by (3.3), we see that $\Phi s=\Phi_{0} \circ \Phi_{1} s=s_{1}$. This implies that $\Phi^{*}$ maps homeomorphically $H_{1}$ onto $H$.

We next show that $H_{1}$ is homeomorphic to $\mathfrak{M}(A(\mathbf{X})) \backslash \mathbf{X}$. When $\xi$ lies in $H_{1}$, we write $\sigma(\xi)$ for the restriction of $\xi$ to $A(\mathbf{X})$. Then $\sigma(\xi)$ cannot lie in $\mathbf{X}$, since $\left|s_{1}(\xi)\right|<1$. Therefore $\sigma$ maps continuously $H_{1}$ into $\mathfrak{M}(A(\mathbf{X})) \backslash \mathbf{X}$. If $\xi_{0}$ lies in $\mathfrak{M}(A(\mathbf{X})) \backslash \mathbf{X}$, then $\left|s_{1}\left(\xi_{0}\right)\right|<1$, since the representing measure for $\xi_{0}$ is quasi-invariant. It follows from Lemma 2.5 that $\sigma$ is a one-to-one map of $H_{1}$ onto $\mathfrak{M}(A(\mathbf{X})) \backslash \mathbf{X}$.

To show the continuity of $\sigma^{-1}$, we take a neighborhood $V$ of $\xi$ in $H_{1}$. Since $H_{1}$ is open in $\mathfrak{M}\left(H^{\infty}(\mathbf{X})\right)$, we notice that $\mathfrak{M}\left(H^{\infty}(\mathbf{X})\right) \backslash V$ is compact in $\mathfrak{M}\left(H^{\infty}(\mathbf{X})\right)$. For any $\eta$ in $\mathfrak{M}\left(H^{\infty}(\mathbf{X})\right) \backslash V$, there is a function $\phi$ in $A(\mathbf{X})$ such that $\phi(\eta)=1$ and $\phi(\xi)=0$. Indeed, if $\eta$ lies in $\mathfrak{M}\left(H^{\infty}(\mathbf{X})\right) \backslash H_{1}$, this follows from the fact that $\left|s_{1}\right|=1$ only on $\mathfrak{M}\left(H^{\infty}(\mathbf{X})\right) \backslash H_{1}$. On the other hand, since $\sigma$ is one-to-one, we see that $A(\mathbf{X})$ separates the points on $H_{1}$. This shows the existence of such a function when $\eta$ lies in $H_{1} \backslash V$. Since $\mathfrak{M}\left(H^{\infty}(\mathbf{X})\right) \backslash V$ 

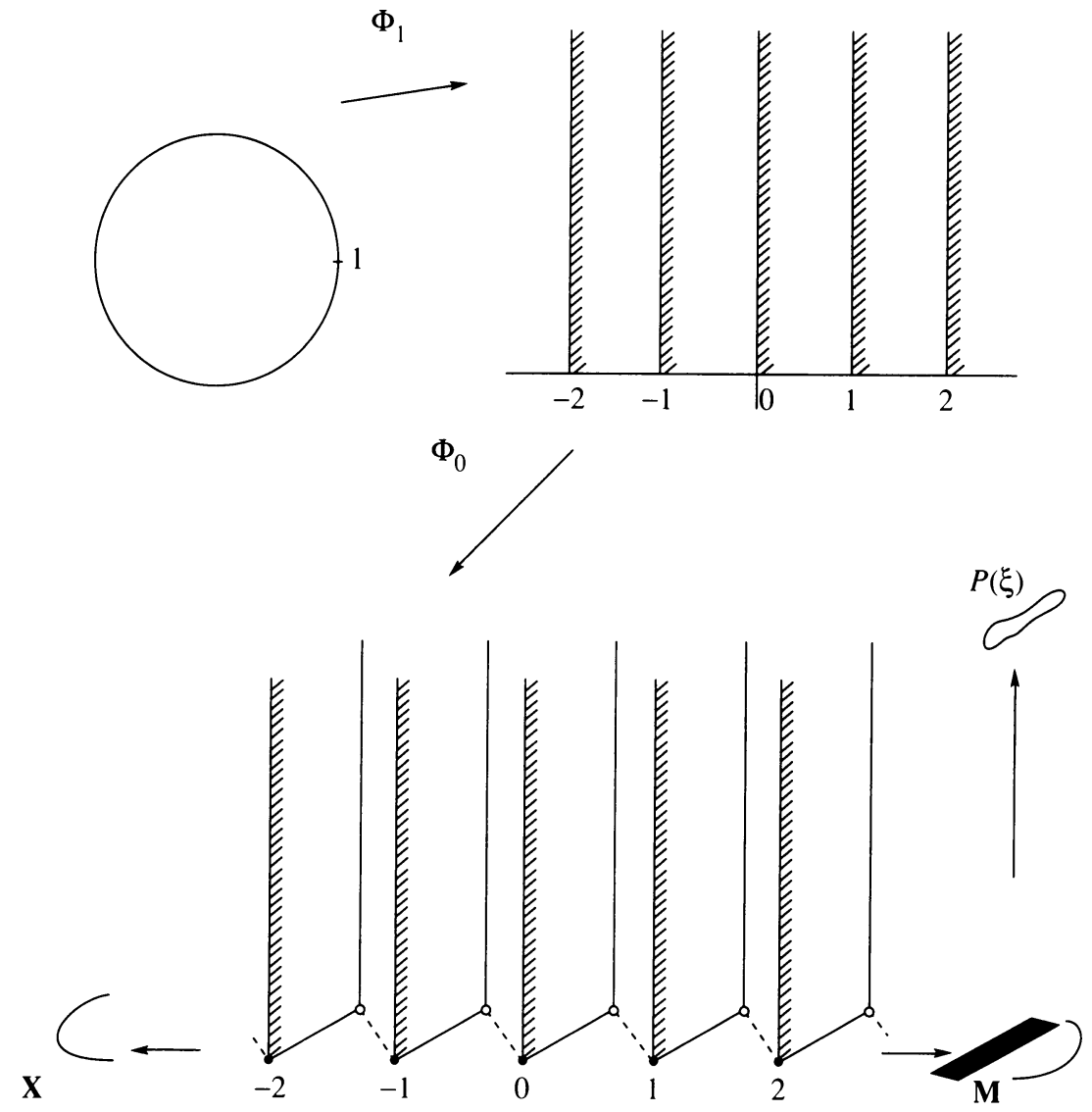

FIGURE 1

is compact, we choose such functions $\phi_{1}, \ldots, \phi_{n}$ in $A(\mathbf{X})$ so that the open subsets $\left\{\left|\phi_{j}\right|>2 / 3\right\}, 1 \leq j \leq n$, make a covering of $\mathfrak{M}\left(H^{\infty}(\mathbf{X})\right) \backslash V$. Define the neighborhood of $\sigma(\xi)$ in $\mathfrak{M}(A(\mathbf{X})) \backslash \mathbf{X}$ by

$$
W=\left\{\eta_{0} \in \mathfrak{M}(A(\mathbf{X})) \backslash \mathbf{X} ;\left|\phi_{j}\left(\eta_{0}\right)\right|<1 / 3,1 \leq j \leq n\right\} .
$$

Then we see $\sigma^{-1} W$ is contained in $V$. Thus $\sigma$ is a homeomorphism.

Let $\pi=\sigma \circ\left(\Phi^{*}\right)^{-1}$. Then $\pi$ maps homeomorphically $H$ onto $\mathfrak{M}(A(\mathbf{X})) \backslash \mathbf{X}$. It is easy to see that $\pi(\Delta)=\mathbf{R}_{+}^{2}$. Since $\mathfrak{M}_{1} \cap H=H \backslash \Delta$, the restriction $\pi_{1}$ maps $\mathfrak{M}_{1} \cap H$ onto $\mathfrak{M}(A(\mathbf{X})) \backslash\left(\mathbf{X} \cup \mathbf{R}_{+}^{2}\right)$. Thus (i) follows.

It follows from (iii) of Lemma 2.4 and Lemma 2.5 that

$$
\|\xi-\eta\|=\|\sigma(\xi)-\sigma(\eta)\|
$$

whenever $\xi$ and $\eta$ lie in $H_{1}$. Since $\Phi^{*}$ is isometric, the map $\pi$ preserves Gleason metrics. We notice that $H$ and $\mathfrak{M}_{1} \cap H$ are unions of Gleason parts.

Suppose that $F$ is an analytic map of $\Delta$ into $H$. If $\phi$ lies in $A(\mathbf{X})$, then $\Phi^{-1} \phi(F(z))$ is analytic on $\Delta$, since $\Phi^{-1} \phi$ lies in $H^{\infty}(\Delta)$. This implies that $\pi \circ F$ is an analytic map of $\Delta$ into $\mathfrak{M}(A(\mathbf{X})) \backslash \mathbf{X}$. Conversely, suppose that $\pi \circ F$ is analytic. Let $\phi$ be a function in $H^{\infty}(\mathbf{X})$. Then $\tilde{\phi}$ denotes the Borel version by Lemma 2.4. Since $\tilde{\phi} * P_{i r}$ is in $A(\mathbf{X})$ for $r>0, \tilde{\phi} * P_{i r}(\pi \circ F(z))$ is analytic 
on $\Delta$. Then we see by (iii) of Lemma 2.4 that

$$
\lim _{r \rightarrow+0} \tilde{\phi} * P_{i r}(\pi \circ F(z))=\phi\left(\left(\Phi^{*}\right)^{-1} \circ F(z)\right),
$$

since the representing measure for $\pi \circ F(z)$ is quasi-invariant on $\mathbf{X}$. This shows that $\Phi^{-1} \phi(F(z))$ is analytic on $\Delta$. Since $\Phi^{-1}$ maps $H^{\infty}(\mathbf{X})$ onto $H^{\infty}(\Delta)$, $F(z)$ is an analytic map of $\Delta$ into $H$. This completes the proof.

Let $\mu$ be an invariant, ergodic, probability measure on $\mathbf{X}$. Then $\mu$ is a representing measure for $A(\mathbf{X})$. As we mentioned earlier, there are many such measures. We identify here $\mu$ with the homomorphism of $A(\mathbf{X})$ by $\mu$. By Theorem 3.1, $\mu$ determines a homomorphism of $H^{\infty}(\Delta)$ lying in $\mathfrak{M}_{1}$. We claim that $\{\mu\}$ is a one-point part in $\mathfrak{M}(A(\mathbf{X}))$. Indeed, since $A(\mathbf{X})$ is a logmodular algebra by Proposition 2.3, each Gleason part is either a one-point part or an analytic disc. Let $H^{2}(\mu)$ be the closure of $A(\mathbf{X})$ in $L^{2}(\mu)$, and let $H_{0}^{2}(\mu)$ be the space of all functions in $H^{2}(\mu)$ which vanish at $\mu$. Then the orthogonal complement of $H_{0}^{2}(\mu) \oplus \overline{H_{0}^{2}}(\mu)$ consists of constants by [11, Theorem I]. Recall that $\mu$ is represented as $\mu_{0} \times m_{I}$, where $\mu_{0}$ is an invariant measure on $\beta \mathbf{Z}$ with respect to $\mathbf{S}$. Suppose $\{\mu\}$ is not a one-point part. Then there is an inner function $q$ in $H^{2}(\mu)$ such that $H_{0}^{2}(\mu)=q H^{2}(\mu)$ (see [5, Chapter V, §7]). Let $q^{\#}(y, t)$ be the automorphic extension of $q$ to $\beta \mathbf{Z} \times \mathbf{R}$. Since the inner function $t \rightarrow q^{\#}(y, t)$ is not constant for $\mu_{0}$-a.e. $y$, we choose easily a nonnegative function $u$ in $L^{\infty}\left(d \mu_{0} \times d t\right)$ satisfying $t \rightarrow q^{\#}(y, t) u(y, t)\left(1+t^{2}\right)^{-1}$ lies in $H^{\infty}(\mathbf{R})$. By setting $g(y, t)=u(y, t)\left(1+t^{2}\right)^{-1}$, a suitable choice of $u$ shows that

$$
\phi^{\#}(y, t)=\sum_{j=-\infty}^{\infty} g\left(\mathbf{S}^{j} y, t-j\right)
$$

for some nonconstant function $\phi$ in $L^{\infty}(\mu)$. Then $\phi$ is orthogonal to $q H^{2}(\mu) \oplus$ $\overline{q H^{2}}(\mu)$, thus we have a contradiction.

The following proposition is, of course, an immediate consequence of the corona theorem, so the point is the direct proof of it.

Proposition 3.2. Let $\mu$ be an invariant, ergodic, probability measure on $\mathbf{X}$. Then the homomorphism in $\mathfrak{M}(A(\mathbf{X}))$ by $\mu$ is an accumulating point of $\mathbf{R}_{+}^{2}$.

Proof. For each $\phi$ in $A(\mathbf{X})$, the individual ergodic theorem shows that

$$
\int_{\mathbf{X}} \phi d \mu=\lim _{T \rightarrow \infty} \frac{1}{2 T} \int_{-T}^{T} \phi(x+t) d t
$$

for $\mu$-a.e. $x$ in $\mathbf{X}$. It follows from Wiener's Tauberian theorem that the right side of (3.5) is equal to $\lim _{r \rightarrow \infty} \phi * P_{i r}(x)$ for each $x$ such that (3.5) holds (see [14, Proof of Lemma 2.6]). By regarding $\mathbf{X} \times(0, \infty)$ as a subset of $\mathfrak{M}(A(\mathbf{X}))$, $\phi * P_{i r}(x)$ is the value of $\phi$ at the point $(x, r)$ by $(2.1)$. Let $\varepsilon>0$, and let $\phi_{1}, \ldots, \phi_{n}$ lie in $A(\mathbf{X})$. Since the union of finite null sets is also null, we find a point $(x, r)$ in $\mathbf{X} \times(0, \infty)$ which belongs to the neighborhood

$$
W=\left\{\eta \in \mathfrak{M}(A(\mathbf{X})) ;\left|\phi_{j}(\eta)-\phi_{j}(\mu)\right|<\varepsilon, 1 \leq j \leq n\right\}
$$

of $\mu$ in $\mathfrak{M}(A(\mathbf{X}))$. Since $\mathbf{R}_{+}^{2}$ is dense in $\mathbf{X} \times(0, \infty)$, there is a point in $\mathbf{R}_{+}^{2}$ lying in $W$. Thus $\mu$ is an accumulating point of $\mathbf{R}_{+}^{2}$.

Let $\left\{\alpha_{n}\right\}$ be an interpolating sequence in $\Delta$. We define

$$
F_{n}(z)=\frac{z+\alpha_{n}}{1+\bar{\alpha}_{n} z}, \quad z \in \Delta,
$$


which is an analytic map of $\Delta$ into $\mathfrak{M}\left(H^{\infty}(\Delta)\right)$, since $\Delta$ is regarded as a subset of $\mathfrak{M}\left(H^{\infty}(\Delta)\right)$. If $F$ is an accumulating point of $\left\{F_{n}\right\}$ in $\mathfrak{M}\left(H^{\infty}(\Delta)\right)^{\Delta}$, then $F$ is an analytic disc. Conversely, all analytic structure in $\mathfrak{M}\left(H^{\infty}(\Delta)\right) \backslash \Delta$ comes about in this manner. This is the characterization of nontrivial parts in $\mathfrak{M}\left(H^{\infty}(\Delta)\right)$ due to Hoffman (see [9] or [6, Chapter X]). We define similarly

$$
L_{n}(z)=\frac{w_{n}-\bar{w}_{n} z}{1-z}, \quad z \in \Delta
$$

for an interpolating sequence $\left\{w_{n}\right\}$ in $\mathbf{R}_{+}^{2}$. Then $L_{n}$ is an analytic map of $\Delta$ into $\mathfrak{M}(A(\mathbf{X}))$ and lies in $[\mathfrak{M}(A(\mathbf{X})) \backslash \mathbf{X}]^{\Delta}$. Suppose that an accumulating point $L$ of $\left\{L_{n}\right\}$ in $\mathfrak{M}(A(\mathbf{X}))^{\Delta}$ lies in $[\mathfrak{M}(A(\mathbf{X})) \backslash \mathbf{X}]^{\Delta}$. It then follows from Hoffman's theorem and Theorem 3.1 that $L$ is an analytic disc in $\mathfrak{M}(A(\mathbf{X}))$. This fact will be used in $\S 4$.

Recall that an interpolating Blaschke product $B(z)$ on $\mathbf{R}_{+}^{2}$ with zeros $\left\{w_{n}=\right.$ $\left.t_{n}+i s_{n}\right\}$ is thin if $B(z)$ satisfies that

$$
\lim _{n \rightarrow \infty} 2 s_{n}\left|B^{\prime}\left(w_{n}\right)\right|=1
$$

Suppose that $\left\{s_{n}\right\}$ is bounded away from zero. Let $\left\{L_{n}\right\}$ and $L$ be as in above. Then $L$ is an analytic disc which is homeomorphic to $\Delta$ and $L^{-1}$ is the product $c B$ of $B$ by a unimodular constant $c$ (see [6, Chapter X, Exercise 8]). From this fact, we obtain the following:

Proposition 3.3. There is a nontrivial part $P$ in $\mathfrak{M}(A(\mathbf{X}))$ satisfying the following properties:

(i) Let $L$ be the analytic map of $\Delta$ onto $P$. Then $L$ is a homeomorphism.

(ii) Let $\mu$ be the representing measure for $L(0)$. Then $\mu$ is invariant on $\mathbf{X}$. Furthermore, there is a measurable function $K(z, x)$ on $\Delta \times \mathbf{X}$ such that $x \rightarrow K(z, x)$ is invariant on $\mathbf{X}$ and

$$
\phi(L(z))=\int_{\mathbf{X}} \phi(x) K(z, x) d \mu(x), \quad \phi \in A(\mathbf{X}) .
$$

Proof. Let us consider the case that $\left\{s_{n}\right\}$ diverges, and let $P=L(\Delta)$. Then we see that the representing measure for $L(z)$ is invariant for each $z$ in $\Delta$. Since $A(\mathbf{X})$ is logmodular, each representing measure for $L(z)$ is mutually absolutely continuous to $\mu$. Let $\theta(x)$ be the invariant function by the right side of $(3.5)$ with $c B$ in place of $\phi$. It is easy to see that the function

$$
K(z, x)=\frac{1}{2 \pi} \frac{1-|z|^{2}}{|\theta(x)-z|^{2}}
$$

satisfies the desired property.

\section{ON A QUESTION OF FORELLI}

Let $\mathscr{C}$ be the class of all closed, nonempty, invariant subsets in a flow $\left(\Omega,\left\{U_{t}\right\}_{t \in \mathbf{R}}\right)$. A set in $\mathscr{C}$ is minimal in $\left(\Omega,\left\{U_{t}\right\}_{t \in \mathbf{R}}\right)$ if it is minimal with respect to the inclusion relation of $\mathscr{C}$. Then Zorn's lemma guarantees the existence of a minimal set. We notice that $\left(\Omega,\left\{U_{t}\right\}_{t \in \mathbf{R}}\right)$ is a minimal flow if and only if $\Omega$ is minimal by itself. A minimal flow is said to be strictly ergodic if there is exactly one invariant probability measure. 
As a generalization of Wermer's maximality theorem [8, Chapter 6], Forelli [4] showed that if $\left(\Omega,\left\{U_{t}\right\}_{t \in \mathbf{R}}\right)$ is minimal, then the induced uniform algebra $A(\Omega)$ is a maximal algebra. In connection with this result, he has asked the question stated in Introduction (see $[12, \S 6]$ for a nice account of related topics). In response to this question, Muhly [11, Theorem II] gave a sufficient condition: If $\left(\Omega,\left\{U_{t}\right\}_{t \in \mathbf{R}}\right)$ is strictly ergodic, then $A(\Omega)$ is a Dirichlet algebra on $\Omega$. Thus, under the condition of strictly ergodicity, many interesting results have been obtained.

Our objective in this section is to give a negative answer to the question:

Theorem 4.1. There exists a minimal flow on which the induced uniform algebra is not a Dirichlet algebra.

Let $\left(\mathbf{X},\left\{\mathbf{S}_{t}\right\}_{t \in \mathbf{R}}\right)$ be the flow in $\S 2$, and let $\mathbf{M}$ be a minimal set in it. We denote the restriction of $\left\{\mathbf{S}_{t}\right\}_{t \in \mathbf{R}}$ to $\mathbf{M}$ by $\left\{T_{t}\right\}_{t \in \mathbf{R}}$. Then $\left(\mathbf{M},\left\{T_{t}\right\}_{t \in \mathbf{R}}\right)$ is a minimal flow (see Figure 1 for the situation). We shall show the induced uniform algebra $A(\mathbf{M})$ is not a Dirichlet algebra on $\mathbf{M}$.

The outline of our proof runs as follows. As usual, $\mathbf{M} \times(0, \infty)$ is regarded as a subset of the maximal ideal space $\mathfrak{M}(A(\mathbf{M}))$ of $A(\mathbf{M})$. For a given $x_{0}$ in $\mathbf{M}$, we choose a suitable divergent sequence $\left\{u_{n}\right\}$ such that an accumulating point $\xi$ of $\left\{\left(x_{0}, u_{n}\right)\right\}$ lies in a nontrivial part $P(\xi)$ that is homeomorphic to $\Delta$. This follows from Hoffman's theorem and Theorem 3.1. Furthermore, by virtue of Marshall's theorem [10], we see that the closure $\overline{P(\xi)}$ of $P(\xi)$ is a homeomorphic replica of $\mathfrak{M}\left(H^{\infty}(\Delta)\right)$ and that the restriction $\left.A\right|_{\overline{P(\xi)}}$ of $A(\mathbf{M})$ to $\overline{P(\xi)}$ is isometrically isomorphic to $H^{\infty}(\Delta)$. Let $\Gamma_{0}$ be the Silov boundary of $\left.A\right|_{\overline{P(\xi)}}$. Since $H^{\infty}(\Delta)$ is not a Dirichlet algebra on its Šilov boundary, a real measure on $\Gamma_{0}$ is orthogonal to $\left.A\right|_{\overline{P(\xi)}}$. Although $\Gamma_{0}$ lies off $\mathbf{M}$, the Riesz representation theorem assures the existence of a real invariant measure on $\mathbf{M}$ which is orthogonal to $A(\mathbf{M})$. Consequently, $A(\mathbf{M})$ cannot be a Dirichlet algebra.

We begin by constructing artificial Blaschke products. Let $N$ be a positive integer, and fix $\gamma=t+i u$ in $\mathbf{R}_{+}^{2}$. We then set

$$
\zeta_{j}=N j+\gamma, \quad j \in \mathbf{Z} .
$$

Then the points $\left\{\zeta_{j}\right\}$ are periodically distributed on the horizontal line $\{\operatorname{Im} z=$ $u\}$ and form an interpolating sequence in $\mathbf{R}_{+}^{2}$. We denote by $B(N, \gamma)(z)$ the Blaschke product with zeros $\left\{\zeta_{j}\right\}$ (see [6, Chapter II, (2.3)]). It is useful to represent $B(n, \gamma)(z)$ by another form. Observe that $z \rightarrow e^{2 \pi i z / N}$ is an inner function in $H^{\infty}\left(\mathbf{R}_{+}^{2}\right)$ with period $N$. Then we have that

$$
B(N, \gamma)(z)=c \frac{e^{2 \pi i z / N}-e^{2 \pi i \gamma / N}}{1-e^{2 \pi i z / N} e^{\overline{2 \pi i \gamma / N}}},
$$

where $c$ is a unimodular constant. In particular, if $u>1$, then we write

$$
z_{j}=N j+i u, \quad j \in \mathbf{Z} \text {. }
$$

Since $z_{j}$ and $z_{-j}$ are axial symmetric with respect to the imaginary axis, $B(N, i u)$ has a simple form

$$
B(N, i u)(z)=-\prod_{j=-\infty}^{\infty} \frac{z-z_{j}}{z-\bar{z}_{j}}=-\frac{z-i u}{z+i u} \prod_{j=1}^{\infty} \frac{(z-i u)^{2}-N^{2} j^{2}}{(z+i u)^{2}-N^{2} j^{2}}
$$


Setting $\gamma=t+i u$, we see that $B(N, \gamma)(z)=c B(N, i u)(z-t)$ for a unimodular constant $c$.

The pseudo-hyperbolic distances $\rho_{1}$ and $\rho$ on $\Delta$ and $\mathbf{R}_{+}^{2}$ are defined by

$$
\begin{cases}\rho_{1}(z, w)=\left|\frac{z-w}{1-\bar{w} z}\right| & \text { on } \Delta, \text { and } \\ \rho(z, w)=\left|\frac{z-w}{z-\bar{w}}\right| & \text { on } \mathbf{R}_{+}^{2},\end{cases}
$$

respectively. Let $L$ be a linear fractional map of $\Delta$ onto $\mathbf{R}_{+}^{2}$. Then we see that $\rho_{1}(z, w)=\rho(L(z), L(w))$ on $\Delta \times \Delta$. Let $0<\lambda<1$, and set

$$
U(w, \lambda)=\left\{z \in \mathbf{R}_{+}^{2} ; \rho(z, w)<\lambda\right\}
$$

for $w=t+i s$ in $\mathbf{R}_{+}^{2}$. Then an easy calculation shows that $U(w, \lambda)$ is an open disc with center $\left(t, s\left(1+\lambda^{2}\right) /\left(1-\lambda^{2}\right)\right)$ and radius $2 s \lambda /\left(1-\lambda^{2}\right)$. It follows from [6, Chapter I, Lemma 1.4] that if $\zeta$ lies in $U(w, \lambda)$, then $U(\zeta, \lambda)$ is contained in $U\left(w, 2 \lambda /\left(1+\lambda^{2}\right)\right)$.

Lemma 4.2. Let $K>0$, and let $0<\delta<\varepsilon<1$. Then there are a positive integer $N=N(K, \varepsilon, \delta)$ and a real number $u=u(K, \varepsilon, \delta), u>1$, satisfying the following properties:

(i) Let $\left\{z_{j}\right\}$ be the sequence by (4.3) with these $N$ and $u$, and let

$$
U_{j}=\left\{z ; \rho\left(z, z_{j}\right)<2(1-\varepsilon) /\left(1+(1-\varepsilon)^{2}\right)\right\}
$$

for each $j$ in $\mathbf{Z}$. Then the discs $\left\{U_{j}\right\}$ are pairwise disjoint, and each $U_{j}$ is contained in the half-plane $\{\operatorname{Im} z>K\}$ (see Figure 2 on the next page).

(ii) Let $\gamma$ in $\mathbf{R}_{+}^{2}$ satisfy that $\rho(\gamma, i u)<1-\varepsilon$, and let $\left\{\zeta_{j}\right\}$ be the sequence by (4.1). If $B(N, \gamma)(z)$ denotes the Blaschke product with zeros $\left\{\zeta_{j}\right\}$, then

$$
|B(N, \gamma)(z)| \geq 1-\delta \text { on }\{0 \leq \operatorname{Im} z \leq K\}
$$

and

$$
|| B(N, \gamma)(z)\left|-\rho\left(z, \zeta_{j}\right)\right|<\varepsilon \quad \text { on } U_{j}
$$

(iii) To each $\delta^{\prime}>0$, there correspond $a=a(\gamma), 1-\delta<a<1$, and $K^{\prime}, K^{\prime}>K$, such that

$$
\sup \left\{|| B(N, \gamma)(z)|-a| ; \operatorname{Im} z>K^{\prime}\right\}<\delta^{\prime},
$$

that is, the left side of (4.8) converges to 0 as $K^{\prime} \rightarrow \infty$. Furthermore, for each square $Q=\left[t_{0}, t_{0}+l\right] \times[0, l], l>K^{\prime}$, the inequality

$$
\sum_{\zeta_{j} \in Q} \operatorname{Im} \zeta_{j} \leq \varepsilon l
$$

holds.

Proof. By setting $\lambda=2(1-\varepsilon) /\left(1+(1-\varepsilon)^{2}\right), U_{j}$ is the disc with center $\left(N j, u\left(1+\lambda^{2}\right) /\left(1-\lambda^{2}\right)\right)$ and radius $2 u \lambda /\left(1-\lambda^{2}\right)$. Thus the discs $\left\{U_{j}\right\}$ have the property (i) whenever $N$ and $u$ satisfy that

$$
u>K(1+\lambda) /(1-\lambda) \text { and } N>4 u \lambda /\left(1-\lambda^{2}\right)
$$




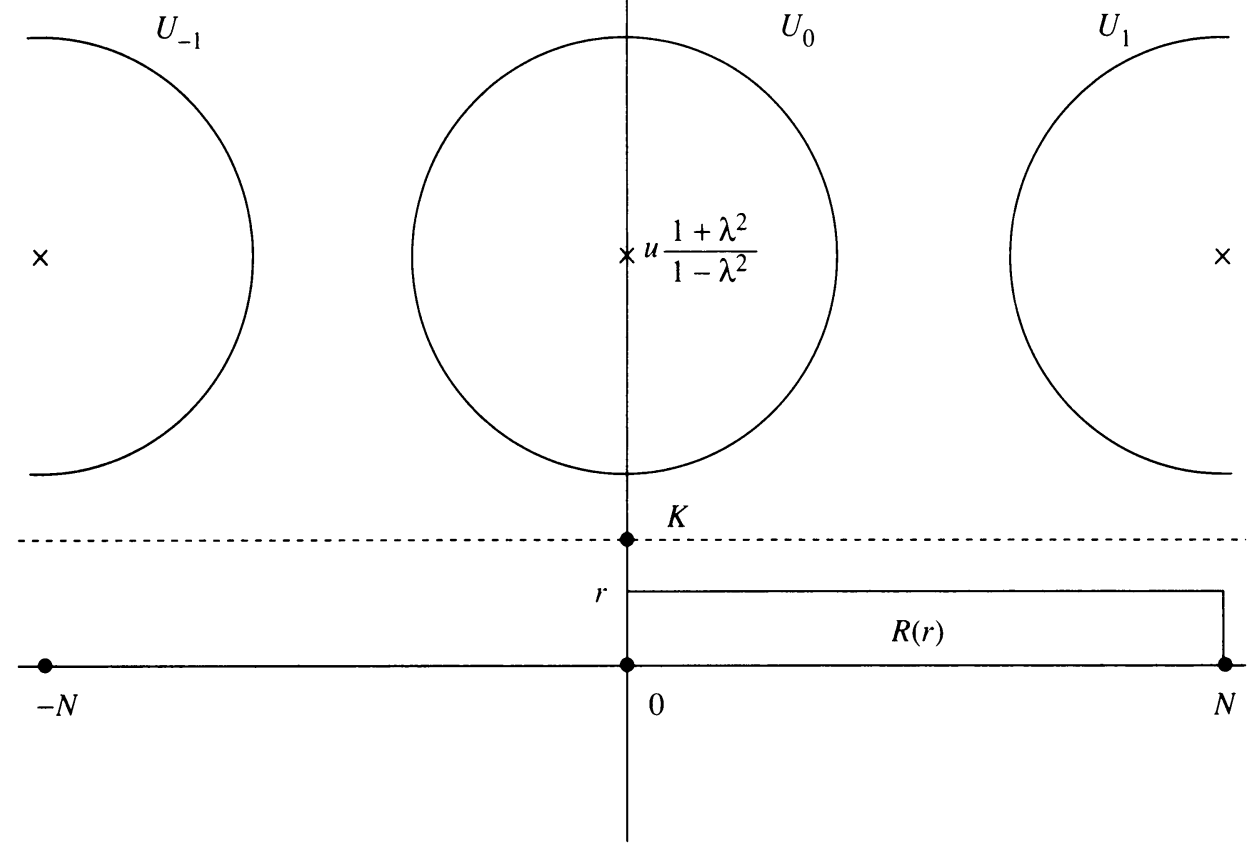

FIGURE 2

Let $(N, u)$ be a pair with the property (i). We notice that $u$ and $N / u$ may be chosen as large as desired. Define that

$$
W_{0}=\{\gamma ; \rho(\gamma, i u) \leq 1-\varepsilon\}
$$

Then $W_{0}$ is a compact disc contained in $U_{0}$. Let $R(r), 0 \leq r \leq K$, be the rectangle $[0, N] \times[0, r]$ (see Figure 2). We claim that $(z, \gamma) \rightarrow|B(N, \gamma)(z)|$ is uniformly continuous on $R(K) \times W_{0}$. Indeed, we find easily a constant $c>0$ such that

$$
1-\rho(z, N j+\gamma)^{2}<c / j^{2}, \quad j \neq 0,
$$

on $R(K) \times W_{0}$. Then the product $|B(N, \gamma)(z)|=\prod_{j=-\infty}^{\infty} \rho(z, N j+\gamma)$ converges uniformly on $R(K) \times W_{0}$. Since each $(z, \gamma) \rightarrow \rho(z, N j+\gamma)$ is continuous on $R(K) \times W_{0}$, the uniform continuity of $|B(N, \gamma)(z)|$ follows from the compactness of $R(K) \times W_{0}$. Consequently, since $|B(N, \gamma)(z)|=1$ on $R(0) \times W_{0}$, there is an $r, 0<r<K$, such that $|B(N, \gamma)(z)|>1-\delta$ on $R(r) \times W_{0}$. Since $\rho(z, N j+\gamma)=\rho\left(z, \zeta_{j}\right)=\rho\left(k z, k \zeta_{j}\right)$ we see that

$$
|B(N, \gamma)(z)|=\prod_{j=-\infty}^{\infty} \rho\left(k z, k \zeta_{j}\right)=|B(k N, k \gamma)(k z)|
$$

for each positive integer $k$. Then $|B(k N, k \gamma)(z)|>1-\delta$ on $[0, k N] \times[0, k r]$. Therefore, if we replace $N$ and $u$ by larger ones, we make $(N, u)$ satisfy $|B(N, \gamma)(z)|>1-\delta$ on $R(K) \times W_{0}$. Since $|B(N, \gamma)(z)|$ is periodic with period $N,(4.6)$ follows. 
We see similarly that $(4.11)$ holds on $U_{0} \times W_{0}$ with a suitable constant $c>0$. Since the partial product $\prod_{j=-m}^{m} \rho(z, N j+\gamma)$ converges uniformly to $|B(N, \gamma)(z)|$ on $U_{0} \times W_{0}$, there is an $m$ such that

$$
\left|\prod_{|j| \geq m+1} \rho(z, N j+\gamma)-1\right|<\varepsilon
$$

on $U_{0} \times W_{0}$. Replacing $N$ with one larger than $m N$, we may assume

$$
|| B(N, \gamma)(z)|-\rho(z, \gamma)|<\varepsilon
$$

on $U_{0} \times W_{0}$. Then (4.7) follows from the periodicity of $|B(N, \gamma)(z)|$. Thus we obtain the property (ii).

We write $\gamma=t+i s$. Since $\rho(\gamma, i u)<1-\varepsilon$, we have easily the inequality,

$$
u \frac{1-(1-\varepsilon)}{1+(1-\varepsilon)}<s<u \frac{1+(1-\varepsilon)}{1-(1-\varepsilon)} \text {. }
$$

Since $N / u$ is chosen as large as desired, we may assume that $e^{-2 \pi s / N}>1-\delta$. Define $a(\gamma)=e^{-2 \pi s / N}$. By (4.2), we see that (4.8) holds for some $K^{\prime}>K$.

If we make $N / u$ and $K^{\prime}$ sufficiently large, (4.9) holds for each square $Q=$ $\left[t_{0}, t_{0}+l\right] \times[0, l], l>K^{\prime}$, so the proof is complete.

Let $\left\{\varepsilon_{n}\right\}$ be a decreasing sequence such that $0<\varepsilon_{n}<1$ and $\sum \varepsilon_{n}<\infty$. We then choose a sequence $\left\{\delta_{n}\right\}, 0<\delta_{n}<1$, such that

$$
\prod_{k=n}^{\infty}\left(1-\delta_{k}\right)>1-\varepsilon_{n}, \quad n \geq 1 \text {. }
$$

For a given $K_{n}>0$, let $N_{n}, u_{n}, \gamma_{n}$, and $a_{n}$ be as in Lemma 4.2 with respect to the above $\varepsilon_{n}$ and $\delta_{n}$. We also set $z_{n j}=N_{n} j+i u_{n}$ and $\zeta_{n j}=N_{n} j+\gamma_{n}$, $j \in \mathbf{Z}$.

By induction, we obtain immediately the following:

Lemma 4.3. There is an increasing sequence $\left\{K_{n}\right\}$ with the following properties:

(i) Let $n \geq 2$. Then

$$
\left|\prod_{k=m}^{n-1}\right| B\left(N_{k}, \gamma_{k}\right)\left(z-t_{k}\right)\left|-\prod_{k=m}^{n-1} a_{k}\right|<\varepsilon_{n}
$$

holds on $\left\{\operatorname{Im} z>K_{n}\right\}$, where $0 \leq t_{k} \leq N_{k}-1$ and $m=1,2, \ldots, n-1$.

(ii) Let $U_{n j}=\left\{z ; \rho\left(z, z_{n j}\right)<2\left(1-\varepsilon_{n}\right) /\left(1+\left(1-\varepsilon_{n}\right)^{2}\right)\right\}$. Then each $U_{n j}$, $j \in \mathbf{Z}$, is contained in $\left\{K_{n}<\operatorname{Im} z<K_{n+1}\right\}$.

(iii) If $Q=\left[t_{0}, t_{0}+l\right] \times[0, l], l>K_{n+1}$, then the inequality

$$
\sum_{\zeta_{n j} \in Q} \operatorname{Im} \zeta_{n j} \leq \varepsilon_{n} l, \quad n \geq 1,
$$

holds.

Recall our notation $\mathbf{M}$ for a minimal set in $\left(\mathbf{X},\left\{\mathbf{S}_{t}\right\}_{t \in \mathbf{R}}\right)$. Identifying $\mathbf{X}$ with $\beta \mathbf{Z} \times[0,1)$, we fix an $x_{0}$ in $\mathbf{M}$ of the form $\left(y_{0}, 0\right)$ in $\beta \mathbf{Z} \times[0,1)$. For each $n \geq 1$, we set

$$
Z_{n}(k)=\left\{N_{n} j+k ; j \in \mathbf{Z}\right\}
$$


where $k=0,1, \ldots, N_{n}-1$. Since $\mathbf{Z}$ is the disjoint union of $Z_{n}(k)$, there is a unique $k_{n}$ such that the closure of $Z_{n}\left(k_{n}\right)$ in $\beta \mathbf{Z}$ contains the above point $y_{0}$. We then define

$$
w_{n j}=z_{n j}+k_{n}=\left(N_{n} j+k_{n}\right)+i u_{n} .
$$

Then $\left\{w_{n j} ; j \in \mathbf{Z}\right\}$ is an interpolating sequence for each $n \geq 1$. We notice that $B\left(N_{n}, k_{n}+i u_{n}\right)(z)$ is the Blaschke product with zeros $\left\{w_{n j} ; j \in \mathbf{Z}\right\}$. Recall that $\mathbf{X} \times[0, \infty)$ is embedded in $\mathfrak{M}(A(\mathbf{X}))$ by $(2.1)$. By identifying $X_{0} \times[0, \infty)$ with $\mathbf{R}_{+}^{2}$, the point $\left(x_{0}, u_{n}\right)=\left(\left(y_{0}, 0\right), u_{n}\right)$ lies in the closure of $\left\{w_{n j} ; j \in\right.$ $\mathbf{Z}\}$ in $\mathfrak{M}(A(\mathbf{X}))$. We notice that $\left(x_{0}, u_{n}\right)$ lies also in $\mathfrak{M}(A(\mathbf{M}))$, since $\mathbf{M} \times$ $[0, \infty)$ is a subset of $\mathfrak{M}(A(\mathbf{M}))$. It will be shown that the accumulating point $\xi$ of $\left\{\left(x_{0}, u_{n}\right) ; n \geq 1\right\}$ in $\mathfrak{M}(A(\mathbf{M}))$ lies in a nontrivial part $P(\xi)$ that is homeomorphic to $\Delta$.

Let us show that $\left\{w_{n j} ; n \geq 1, j \in \mathbf{Z}\right\}$ is an interpolating sequence in $\mathbf{R}_{+}^{2}$. These points are clearly separated by (ii) of Lemma 4.3. Since $\sum \varepsilon_{n}<\infty$, it follows from (iii) of Lemma 4.3 that

$$
\sum_{w_{n j} \in Q} \operatorname{Im} w_{n j} \leq\left(1+\sum \varepsilon_{n}\right) l
$$

for each square $Q=\left[t_{0}, t_{0}+l\right] \times[0, l]$. Therefore, by [6, Chapter VII, Theorem 1.1], $\left\{w_{n j} ; n \geq 1, j \in \mathbf{Z}\right\}$ is an interpolating sequence.

Let $B_{m}(z)$ be the Blaschke product with zeros $\left\{w_{n j} ; n \geq m, j \in \mathbf{Z}\right\}$. Since

$$
B_{m}(z)=\prod_{n=m}^{\infty} B\left(N_{n}, k_{n}+i u_{n}\right)(z),
$$

$B_{m}(z)$ has the periodic zeros $\left\{w_{n j} ; j \in \mathbf{Z}\right\}$ on each horizontal line $\{\operatorname{Im} z=$ $\left.u_{n}\right\}, n \geq m$, (see Figure 3 for the distribution of zeros of $\left.B_{1}(z)\right)$. We see

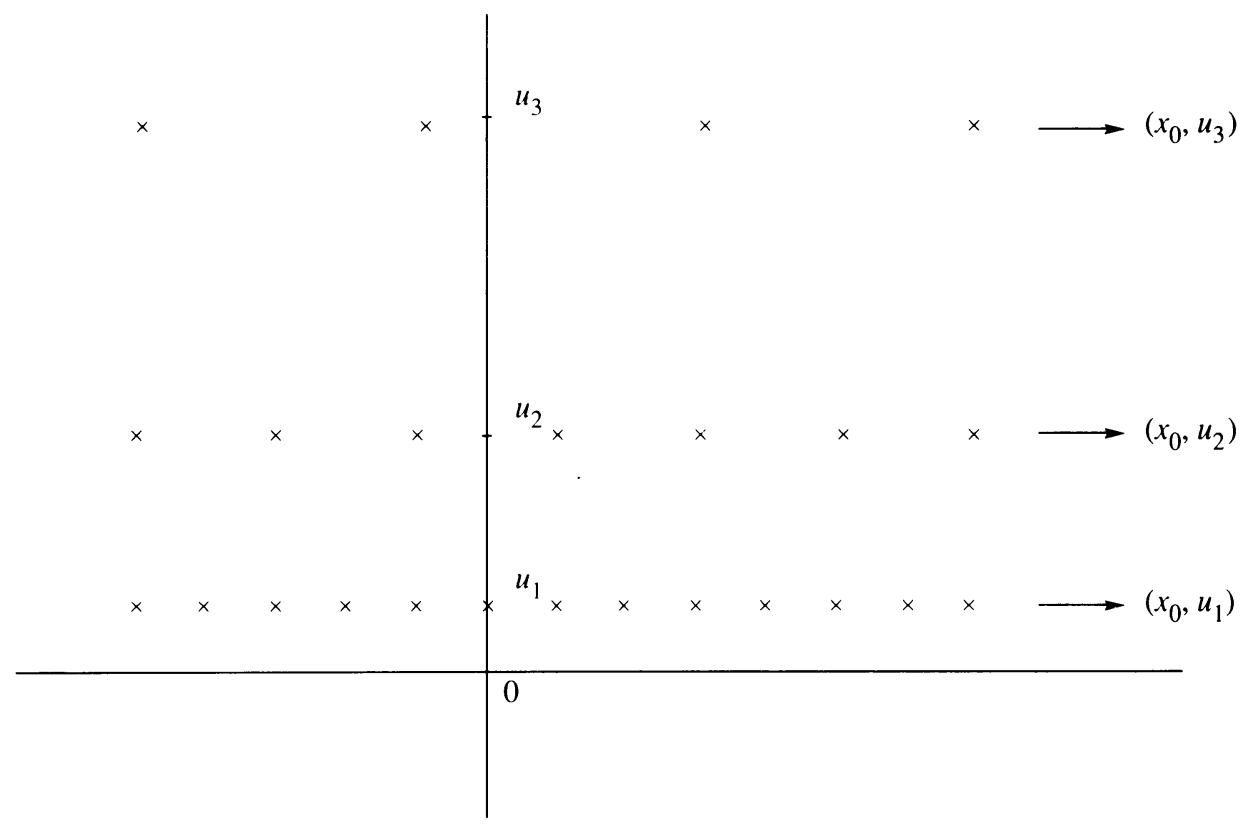

FigURE 3 
by (4.16) and [6, Chapter II, Theorem 6.1] that $B_{m}(z)$ extends analytically to $\left\{\operatorname{Im} z \geq-K_{m}\right\}$. It follows from (4.12) and (ii) of Lemma 4.2 that $\left|B_{m}(z)\right| \leq$ $\left(1-\varepsilon_{m}\right)^{-1}$ on $\left\{\operatorname{Im} z \geq-K_{m}\right\}$. Let $0<s<K_{m}$, and define the bounded function $F$ on $\mathbf{R}$ by $F(t)=B_{m}(t-i s)$. Since $B_{m}(t)=F * P_{i s}(t)$, we see that $t \rightarrow B_{m}(t)$ is uniformly continuous on $\mathbf{R}$. Hence Lemma 3.1 implies that $B_{m}(t)$ extends to a function $B_{m}(x)$ in $A(\mathbf{X})$. Recall that $O\left(x_{0}\right) \times(0, \infty)$ is a nontrivial part in $\mathfrak{M}(A(\mathbf{X}))$. However, since $x_{0}$ is in the minimal set $\mathbf{M}$, $O\left(x_{0}\right) \times(0, \infty)$ is not homeomorphic to $\mathbf{R}_{+}^{2}$. On the other hand, $O\left(x_{0}\right) \times(0, \infty)$ contains every $\left(x_{0}, u_{n}\right)$, which is an accumulating point of $\left\{w_{n j} ; j \in \mathbf{Z}\right\}$.

Let $\left\{\varepsilon_{n}\right\},\left\{\gamma_{n}\right\}$, and $\left\{U_{n j}\right\}$ be as in Lemma 4.3, and let $\left\{w_{n j}\right\}$ be the sequence defined by (4.14). We then set

$$
\begin{aligned}
V_{n j} & =\left\{z ; \rho\left(z, w_{n j}\right)<2\left(1-\varepsilon_{n}\right) /\left(1+\left(1-\varepsilon_{n}\right)^{2}\right)\right\} \\
& =U_{n j}+k_{n} .
\end{aligned}
$$

Observe that $B\left(N_{n}, \gamma_{n}\right)\left(z-k_{n}\right)$ is (a unimodular constant multiple of) the interpolating Blaschke product with zeros $\left\{\zeta_{n j}+k_{n} ; j \in \mathbf{Z}\right\}$, where $\zeta_{n j}=$ $N_{n} j+\gamma_{n}$. By (iii) of Lemma 4.3, (4.15) holds with $\zeta_{n j}+k_{n}$ in place of $w_{n j}$. Then $\left\{\zeta_{n j}+k_{n} ; n \geq 1, j \in \mathbf{Z}\right\}$ is also an interpolating sequence. Let $C_{m}(z)$ be the Blaschke product with zeros $\left\{\zeta_{n j}+k_{n} ; n \geq m, j \in \mathbf{Z}\right\}$. So we may write

$$
C_{m}(z)=\prod_{n=m}^{\infty} B\left(N_{n}, k_{n}+\gamma_{n}\right)(z)
$$

(compare with (4.16)).

Let $\left\{\delta_{n}\right\},\left\{K_{n}\right\}$, and $\left\{a_{n}\right\}$ be also as in Lemma 4.3. Then we see that $\left|B\left(N_{n}, k_{n}+\gamma_{n}\right)(z)\right|>1-\delta_{n}$ on $\left\{0<\operatorname{Im} z<K_{n}\right\}$ and $1-\delta_{n}<a_{n}<1$ by Lemma 4.2. It follows from (4.12) that the product

$$
a^{(m)}=\prod_{n=m}^{\infty} a_{n}
$$

converges and satisfies that $1-\varepsilon_{m}<a^{(m)}<1$.

Lemma 4.4. Let $V_{n j}$ and $C_{m}(z)$ be as in above. Then

$$
\sup \left\{|| C_{m}(z)\left|-a^{(m)} \rho\left(z, \zeta_{n j}+k_{n}\right)\right| ; z \in V_{n j}\right\}
$$

converges to 0 as $n \rightarrow \infty$.

Proof. We set, at our convenience, that

$$
E_{n}(z)=\left|B\left(N_{n}, k_{n}+\gamma_{n}\right)(z)\right|,
$$

and

$$
F_{n}(z)=a^{(n+1)} E_{n}(z) \prod_{k=m}^{n-1} E_{k}(z) .
$$

Then $\left|C_{m}(z)\right|=\prod_{k=m}^{\infty} E_{k}(z)$ by (4.17). Since $V_{n j}$ is contained in $\left\{K_{n}<\right.$ $\left.\operatorname{Im} z<K_{n+1}\right\}$ by (ii) of Lemma 4.3, it follows from (4.6) that

$$
\prod_{k=n+1}^{\infty}\left(1-\delta_{n}\right)<\prod_{k=n+1}^{\infty} E_{k}(z)<1, \quad z \in V_{n j}
$$


Observe that

$$
\left|F_{n}(z)-a^{(n+1)} \rho\left(z, \zeta_{n j}+k_{n}\right) \prod_{k=m}^{n-1} E_{k}(z)\right| \leq\left|E_{n}(z)-\rho\left(z, \zeta_{n j}+k_{n}\right)\right| .
$$

Then we see by (4.7), (4.13), and (4.18) that

$$
\begin{aligned}
& || C_{m}(z)\left|-a_{n}^{-1} a^{(m)} \rho\left(z, \zeta_{n j}+k_{n}\right)\right| \\
& \quad \leq|| C_{m}(z)\left|-F_{n}(z)\right|+\left|F_{n}(z)-a^{(n+1)} \rho\left(z, \zeta_{n j}+k_{n}\right) \prod_{k=m}^{n-1} a_{k}\right| \\
& \quad \leq|| C_{n+1}(z)\left|-a^{(n+1)}\right|+\left|E_{n}(z)-\rho\left(z, \zeta_{n j}+k_{n}\right)\right|+\left|\prod_{k=m}^{n-1} E_{k}(z)-\prod_{k=m}^{n-1} a_{k}\right| \\
& \quad<\left(1-\prod_{k=n+1}^{\infty}\left(1-\delta_{k}\right)\right)+\varepsilon_{n}+\varepsilon_{n} \\
& \quad<3 \varepsilon_{n}
\end{aligned}
$$

for each $z$ in $V_{n j}$. Since $a_{n}^{-1}$ tends to 1 as $n \rightarrow \infty$, the conclusion follows.

We denote by $\xi_{n}$ the homomorphism $\left(x_{0}, u_{n}\right)$ in $\mathfrak{M}(A(\mathbf{X}))$. To each $n \geq$ 1 , there corresponds a subnet $\left\{w_{n \beta}\right\}$ of $\left\{w_{n j} ; j \in \mathbf{Z}\right\}$ converging to $\xi_{n}$ in $\mathfrak{M}(A(\mathbf{X}))$. Let $\xi$ be an accumulating point of $\left\{\xi_{n}\right\}$. Then $\xi$ is also an accumulating point of the interpolating sequence $\left\{w_{n j} ; n \geq 1, j \in \mathbf{Z}\right\}$. Therefore, by Hoffman's theorem and Theorem 3.1, $\xi$ belongs to a nontrivial part $P(\xi)$. Precisely, let $L_{n j}$ be the linear fractional map of $\Delta$ onto $\mathbf{R}_{+}^{2}$ by

$$
L_{n j}(z)=\frac{w_{n j}-\bar{w}_{n j} z}{1-z}, \quad z \in \Delta
$$

Then $L_{n j}$ is an analytic map of $\Delta$ into $\mathfrak{M}(A(\mathbf{X}))$. Taking a finer subnet $\left\{L_{n \beta}\right\}$ of $\left\{L_{n j}\right\}$, if necessary, we may assume that

$$
\lim _{\beta} L_{n \beta}=L_{n}, \quad n \geq 1,
$$

in $\mathfrak{M}(A(\mathbf{X}))^{\Delta}$, where $L_{n}$ is in $[\mathfrak{M}(A(\mathbf{X})) \backslash \mathbf{X}]^{\Delta}$ and satisfies that $L_{n}(0)=\xi_{n}$. Let us determine the explicit form of $L_{n}$. For simplicity, we write $x+t+i s$ for $(x+t, s)$ in $\mathbf{X} \times[0, \infty)$. It then follows from (4.19) that

$$
L_{n}(z)=x_{0}+\frac{i u_{n}(1+z)}{1-z}, \quad z \in \Delta
$$

which maps $\Delta$ onto the nontrivial part $O\left(x_{0}\right) \times(0, \infty)$. We notice that $L_{n \beta}$ and $L_{n}$ lie in $[\mathfrak{M}(A(\mathbf{X})) \backslash \mathbf{X}]^{\Delta}$, so the convergence in (4.20) is the same one in $\mathfrak{M}\left(H^{\infty}(\mathbf{X})\right)^{\Delta}$ by Theorem 3.1. As we mentioned earlier, $L_{n}$ is not a homeomorphism, since $x_{0}$ lies in the minimal set $\mathbf{M}$. We take again a subnet $\left\{L_{\alpha}\right\}$ of $\left\{L_{n}\right\}$ such that

$$
\lim _{\alpha} L_{\alpha}=L_{0}
$$

for a map $L_{0}$ in $\mathfrak{M}(A(\mathbf{X}))^{\Delta}$ with $L_{0}(0)=\xi$. Since $\lim _{\alpha} u_{\alpha}=\infty$, we see by (4.21) that $L_{0}$ is also in $[\mathfrak{M}(A(\mathbf{X})) \backslash \mathbf{X}]^{\Delta}$. It then follows from Theorem 3.1 and Hoffman's theorem that $L_{0}$ is an analytic map and the range $L_{0}(\Delta)$ is the 
nontrivial part $P(\xi)$ containing $\xi$. We recall that the meaning of (4.22) is that if $z$ lies in $\Delta$, then

$$
\lim _{\alpha} \phi \circ L_{\alpha}(z)=\phi \circ L_{0}(z)
$$

for each $\phi$ in $A(\mathbf{X})$.

We next show that $L_{0}$ may be considered as an analytic map of $\Delta$ into $\mathfrak{M}(A(\mathbf{M}))$. Let $E_{\mathbf{X}}$ be the closure of $\mathbf{M} \times[0, \infty)$ in $\mathfrak{M}(A(\mathbf{X}))$. Since $L_{\alpha}(z)$, $z \in \Delta$, is in $\mathbf{M} \times[0, \infty)$ by $(4.21)$ and $\lim _{\alpha} u_{\alpha}=\infty$, we see that $L_{0}(z)$ lies in $E_{\mathbf{X}} \backslash \mathbf{M} \times[0, \infty)$. Then the whole $P(\xi)$ is contained in $E_{\mathbf{X}} \backslash \mathbf{M} \times[0, \infty)$. On the other hand, $\mathbf{M} \times[0, \infty)$ is also considered as a subset of $\mathfrak{M}(A(\mathbf{M}))$. We denote similarly by $E_{\mathbf{M}}$ the closure of $\mathbf{M} \times[0, \infty)$ in $\mathfrak{M}(A(\mathbf{M}))$. We have to examine the relation between $E_{\mathbf{X}}$ and $E_{\mathbf{M}}$.

Let $\left.A\right|_{\mathbf{M}}$ be the space of the restrictions to $\mathbf{M}$ of functions in $A(\mathbf{X})$. Then $\left.{ }^{4}\right|_{\mathbf{M}}$ is a subalgebra of $A(\mathbf{M})$ containing constants. So there is a natural restriction map $\sigma_{1}$ of $E_{\mathbf{M}}$ to $E_{\mathbf{X}}$ which is the identity map on $\mathbf{M} \times[0, \infty)$. Let us show that $\sigma_{1}$ is a homeomorphism of $E_{\mathbf{M}}$ onto $E_{\mathbf{X}}$. Since $A(\mathbf{X})$ is a logmodular algebra, $\sigma_{1}$ is a one-to-one continuous map. If $\eta$ lies in $E_{\mathbf{X}}$, then there is a net $\left\{\eta_{\beta}\right\}$ in $\mathbf{M} \times[0, \infty)$ converging to $\eta$ in $E_{\mathbf{X}}$. Since $E_{\mathbf{M}}$ is compact, we may assume that $\left\{\eta_{\beta}\right\}$ converges to $\eta_{1}$ in $E_{\mathbf{M}}$. We then have that $\sigma_{1}\left(\eta_{1}\right)=\eta$. Since $E_{\mathbf{M}}$ is compact, $E_{\mathbf{M}}$ and $E_{\mathbf{X}}$ are homeomorphic via the map $\sigma_{1}$.

We see that the closure of $\left.A\right|_{\mathbf{M}}$ is a logmodular algebra on $\mathbf{M}$, then so is $A(\mathbf{M})$. If $\sigma_{1}\left(\eta_{1}\right)=\eta$, then $\eta_{1}$ and $\eta$ have the same representing measures. Identifying $L_{0}$ with $\sigma_{1}^{-1} \circ L_{0}$, we see that (4.23) holds for each $\phi$ in $A(\mathbf{M})$. This implies that $L_{0}$ is an analytic map of $\Delta$ into $\mathfrak{M}(A(\mathbf{M}))$.

Since the representing measures for the points in $P(\xi)$ are mutually absolutely continuous, $P(\xi)$ is also a nontrivial part with respect to $A(\mathbf{M})$ by [5, Chapter VI, §1.2].

Let $\mu$ be the representing measure for a point $\eta$ in $E_{\mathbf{M}} \backslash \mathbf{M} \times[0, \infty)$. We show that $\mu$ is invariant on $\left(\mathbf{M},\left\{T_{t}\right\}_{t \in \mathbf{R}}\right)$, although the similar fact has been used tacitly in the proofs of Lemma 2.5 and (ii) of Proposition 3.3. Indeed, there is a net $\left(x_{\beta}, u_{\beta}\right)$ in $\mathbf{M} \times[0, \infty)$ converging to $\eta$, where $\lim _{\beta} u_{\beta}=\infty$. Let $\mu_{\beta}$ be the representing measure for $\left(x_{\beta}, u_{\beta}\right)$. Then $\left\{\mu_{\beta}\right\}$ converges to $\mu$ in the weak-* topology on $M(\mathbf{M})$. Since

$$
\left|P_{i u}(t-s)-P_{i u}(t)\right| \leq \frac{s}{u}\left(1+\frac{s}{u}\right) P_{i u}(t-s),
$$

we see that

$$
\int_{\mathbf{M}} \phi(x+s) d \mu(x)=\int_{\mathbf{M}} \phi(x) d \mu(x), \quad \phi \in C(\mathbf{M}),
$$

for each $s$ in $\mathbf{R}$. Then $\mu$ is invariant on $\mathbf{M}$. In particular, the representing measure for each point in $P(\xi)$ is invariant on $\mathbf{M}$.

Lemma 4.5. Let $\xi_{n}$ denote the homomorphism $\left(x_{0}, u_{n}\right)$ defined above, and let $\xi$ be an accumulating point of $\left\{\xi_{n}\right\}$ in $\mathfrak{M}(A(\mathbf{M}))$. Then $\xi$ lies in a nontrivial part $P(\xi)$ in $\mathfrak{M}(A(\mathbf{M}))$ satisfying the following properties:

(i) Let $\overline{P(\xi)}$ be the closure of $P(\xi)$ in $\mathfrak{M}(A(\mathbf{M}))$. Then each point in $\overline{P(\xi)}$ has a unique representing measure which is invariant on $\mathbf{M}$. 
(ii) The part $P(\xi)$ is homeomorphic to $\Delta$. Precisely, the analytic map $L_{0} b y$ (4.22) has its continuous inverse.

Proof. We have shown that $\xi$ lies in a nontrivial part $P(\xi)$, in which every representing measure is invariant on $\mathbf{M}$. Since the set of all invariant probability measures on $\mathbf{M}$ is weak-* compact in $M(\mathbf{M})$, each point in $\overline{P(\xi)}$ has also an invariant representing measure. Thus (i) follows.

Let $L_{n j}, L_{n}$, and $L_{0}$ be as in (4.19), (4.21), and (4.22), respectively. Let $B_{m}(z), m \geq 1$, be the Blaschke product defined by (4.16). Since $B_{m}(z)$ extends to a function $B_{m}(x)$ in $A(\mathbf{X})$, we may regard $B_{m}(x)$ as a function in $A(\mathbf{M})$ by identifying with its restriction to $\mathbf{M}$. Since

$$
\rho\left(L_{n j}(z), L_{n j}(0)\right)=\rho_{1}(z, 0)=|z|, \quad z \in \Delta,
$$

by (4.5), Lemma 4.4 implies that

$$
\sup \left\{|| B_{m} \circ L_{n j}(z)\left|-a^{(m)}\right| z|| ;|z| \leq 1-\varepsilon_{n}\right\}
$$

converges to 0 as $n \rightarrow \infty$, where $a^{(m)}$ is the constant by (4.18). By (4.20) and (4.22), we obtain that $\left|B_{m} \circ L_{0}(z)\right|=a^{(m)}|z|$ on $\Delta$. It then follows from Schwarz's lemma that $\left(c_{m} B_{m}\right) \circ L_{0}(z)=z$ for a constant $c_{m}$ with $\left|c_{m}\right|=$ $\left(a^{(m)}\right)^{-1}$. Since $B_{m}$ is continuous on $\mathfrak{M}(A(\mathbf{M})), L_{0}$ has the continuous inverse.

To extend (ii) of Lemma 4.5 (see Lemma 4.8), we need the following:

Lemma 4.6. Let $L_{0}$ be the analytic map of $\Delta$ into $\mathfrak{M}(A(\mathbf{X}))$ by (4.22), and let $b(z)$ be an arbitrary Blaschke product on $\Delta$ with zeros $\left\{\alpha_{k}\right\}$, that is,

$$
b(z)=\prod_{k=1}^{\infty} \frac{\bar{\alpha}_{k}}{\left|\alpha_{k}\right|} \frac{\alpha_{k}-z}{1-\bar{\alpha}_{k} z}
$$

(we consider $\overline{\alpha_{k}} /\left|\alpha_{k}\right|=-1$, if $\alpha_{k}=0$ ). If $0<\varepsilon<1$, then there is a Blaschke product $B(z)$ on $\mathbf{R}_{+}^{2}$ such that $B(z)$ extends to a function $B(x)$ in $A(\mathbf{X})$ satisfying that

$$
c B \circ L_{0}(z)=b(z), \quad z \in \Delta,
$$

for some constant $c$ with $|c|<1+\varepsilon$.

Proof. We have already done the hard work back in Lemma 4.4. Let $\left\{\varepsilon_{n}\right\}$ and $\left\{\delta_{n}\right\}$ be as in (4.12), and let $\left\{r_{k}\right\}$ be a sequence such that $0<r_{k}<1$ and $\prod r_{k}>(1+\varepsilon)^{-1}$. We assume that $\left|\alpha_{k}\right| \leq\left|\alpha_{k+1}\right|$ for all $k \geq 1$.

Let $w_{n j}$ and $L_{n j}$ be as in (4.14) and (4.19), respectively. Then we see by (4.5) that $\rho_{1}(z, w)=\rho\left(L_{n j}(z), L_{n j}(w)\right)$ on $\Delta \times \Delta$. Since $L_{n j}(0)=w_{n j}$, to each $k \geq 1$, there corresponds $m_{1}(k)$ such that

$$
\rho\left(w_{n j}, L_{n j}\left(\alpha_{k}\right)\right)=\rho_{1}\left(0, \alpha_{k}\right)=\left|\alpha_{k}\right|<1-\varepsilon_{n}
$$

for all $n \geq m_{1}(k)$. Observe that $L_{n j}\left(\alpha_{k}\right)=N_{n} j+L_{n 0}\left(\alpha_{k}\right)$ by (4.19). By (iii) of Lemma 4.3, $\left\{L_{n j}\left(\alpha_{k}\right) ; n \geq m_{1}(k), j \in \mathbf{Z}\right\}$ is an interpolating sequence in $\mathbf{R}_{+}^{2}$. Making $m_{1}(k)$ large, we may assume that

$$
\sum_{n=m_{1}(k)}^{\infty} \sum_{j=-\infty}^{\infty} \frac{\operatorname{Im} L_{n j}\left(\alpha_{k}\right)}{1+\left|L_{n j}\left(\alpha_{k}\right)\right|^{2}}<\left(\frac{1}{2}\right)^{k} .
$$


Let $m(k) \geq m_{1}(k)$. We denote by $C_{m(k)}(z)$ the Blaschke product on $\mathbf{R}_{+}^{2}$ with zeros $\left\{L_{n j}\left(\alpha_{k}\right) ; n \geq m(k), j \in \mathbf{Z}\right\}$. Then $C_{m(k)}(z)$ is the Blaschke product in (4.17) with $L_{n 0}\left(\alpha_{k}\right)$ in place of $\gamma_{n}$. Let $a^{(m(k))}$ be the constant by (4.18) with respect to $C_{m(k)}(z)$. If we make $m(k)$ sufficiently large, then $a^{(m(k))}$ satisfies that $r_{k}<a^{(m(k))}<1$. By Lemma 4.4, we see that

$$
\sup \left\{|| C_{m(k)}(z)\left|-a^{(m(k))} \rho\left(z, L_{n j}\left(\alpha_{k}\right)\right)\right| ; z \in V_{n j}\right\}
$$

converges to 0 as $n \rightarrow \infty$. By induction, we choose an increasing sequence $\{m(k)\}, m(k) \geq m_{1}(k)$, such that

$$
\sup \left\{|| \prod_{i=1}^{k} C_{m(i)}(z)\left|-\prod_{i=1}^{k} a^{(m(i))} \rho\left(z, L_{n j}\left(\alpha_{i}\right)\right)\right| ; z \in V_{n j}\right\}<\varepsilon_{k}
$$

for each $n$ with $m(k+1) \geq n>m(k)$, and that

$$
\left|C_{m(k)}(z)\right| \geq \prod_{i=m(k)}^{\infty}\left(1-\delta_{i}\right)>1-\delta_{k}
$$

on $\left\{0 \leq \operatorname{Im} z<K_{m(k)}\right\}$ by (4.6) and (4.17). We then set

$$
B(z)=\prod_{i=1}^{\infty} C_{m(i)}(z) \text { and } a=\prod_{i=1}^{\infty} a^{(m(i))} .
$$

If $m(k+1) \geq n>m(k)$, then we have by (4.12) that

$$
|| B(z)|-| \prod_{i=1}^{k} C_{m(i)}(z)|| \leq|| \prod_{i=k+1}^{\infty} C_{m(i)}(z)|-1|<1-\prod_{i=k+1}^{\infty}\left(1-\delta_{i}\right)<\varepsilon_{k}
$$

on $V_{n j}$. This implies that

$$
\sup \left\{|| B(z)\left|-\prod_{i=1}^{k} a^{(m(i))} \rho\left(z, L_{n j}\left(\alpha_{i}\right)\right)\right| ; z \in V_{n j}\right\}<2 \varepsilon_{k} .
$$

Let $b_{k}(z)$ be the partial product of $b(z)$ :

$$
b_{k}(z)=\prod_{i=1}^{k} \frac{\bar{\alpha}_{i}}{\left|\alpha_{i}\right|} \frac{\alpha_{i}-z}{1-\bar{\alpha}_{i} z} .
$$

Then, whenever $m(k+1) \geq n>m(k)$, we obtain that

$$
\sup \left\{|| B \circ L_{n j}(z)\left|-\prod_{i=1}^{k} a^{(m(i))}\right| b_{k}(z)|| ;|z| \leq 1-\varepsilon_{n}\right\}<2 \varepsilon_{k} .
$$

Consequently, it follows from (4.20) and (4.22) that

$$
\left|B \circ L_{0}(z)\right|=a|b(z)|, \quad z \in \Delta .
$$

Since $a>(1+\varepsilon)^{-1}$, the maximum principle shows that (4.25) holds for a constant $c$ with $|c|=a^{-1}$.

Recall that we identify $\phi$ in $A(\mathbf{M})$ with its Gelfand transform on $\mathfrak{M}(A(\mathbf{M}))$. Then $\left.\phi\right|_{P(\xi)}$ denotes the restriction of $\phi$ to $P(\xi)$. Let $\left.A\right|_{P(\xi)}$ denote the algebra 
of all $\left.\phi\right|_{P(\xi)}$ for $\phi$ in $A(\mathbf{M})$. Then the analytic map $L_{0}$ in (4.22) induces naturally the isometric isomorphism $\Psi$ of $\left.A\right|_{P(\xi)}$ to $H^{\infty}(\Delta)$ by

$$
\Psi\left(\left.\phi\right|_{P(\xi)}\right)(z)=\phi \circ L_{0}(z), \quad z \in \Delta .
$$

If $b(z)$ is a Blaschke product, let us agree to also call $c b(z)$ a Blaschke product when $c$ is a unimodular constant. Together with Bernard's trick, Marshall has shown that the unit ball of $H^{\infty}(\Delta)$ is the norm closed convex hull of the set of Blaschke products (see [10] or [6, Chapter V, Corollary 2.6]).

Lemma 4.7. Let $f$ be a function in $H^{\infty}(\Delta)$ with $\|f\|_{\infty}=1$, and let $\varepsilon>0$. Then there is a function $\psi$ in $A(\mathbf{M})$ with $\|\psi\|_{\infty}<1+\varepsilon$ such that $\psi \circ L_{0}(z)=$ $f(z)$ on $\Delta$. Consequently, the isomorphism $\Psi$ by (4.28) maps $\left.A\right|_{P(\xi)}$ onto $H^{\infty}(\Delta)$.

Proof. It follows from Marshall's theorem that there is a sequence $\left\{q_{n}\right\}$ of convex combinations of Blaschke products on $\Delta$ such that

$$
f=q_{0}+\frac{\varepsilon}{3} \sum_{n=1}^{\infty}\left(\frac{1}{2}\right)^{n} q_{n}
$$

On the other hand, Lemma 4.6 shows that we may choose a convex combination $Q_{n}$ of Blaschke products on $\mathbf{R}_{+}^{2}$ which extends to a function in $A(\mathbf{X})$ such that $(1+\varepsilon / 3) Q_{n} \circ L_{0}=q_{n}, n \geq 0$. Since $\left\|Q_{n}\right\|_{\infty} \leq 1$, the series

$$
\psi(x)=\left(1+\frac{\varepsilon}{3}\right)\left(Q_{0}(x)+\frac{\varepsilon}{3} \sum_{n=1}^{\infty}\left(\frac{1}{2}\right)^{n} Q_{n}(x)\right)
$$

converges uniformly on $\mathbf{X}$, and belongs to $A(\mathbf{X})$. Of course, the restriction of $\psi$ to $\mathbf{M}$ lies in $A(\mathbf{M})$. It is easy to see that $\|\psi\|_{\infty}<1+\varepsilon$ and $\psi \circ L_{0}(z)=f(z)$ on $\Delta$.

Let $\left.A\right|_{\overline{P(\xi)}}$ similarly be the algebra of all restrictions to the closure $\overline{P(\xi)}$ of functions in $A(\mathbf{M})$, and let $\widehat{H}^{\infty}(\Delta)$ be the uniform algebra on $\mathfrak{M}\left(H^{\infty}(\Delta)\right)$ of all Gelfand transforms of functions in $H^{\infty}(\Delta)$.

The following lemma is an easy consequence of the corona theorem and Lemma 4.7, so the proof is omitted.

Lemma 4.8. Let $\Gamma$ and $\Gamma_{0}$ be the Šilov boundaries of $\hat{H}^{\infty}(\Delta)$ and $\left.A\right|_{\overline{P(\xi)}}$, respectively. Let $L_{0}$ be the analytic map of $\Delta$ onto $P(\xi)$ by (4.22). Then $L_{0}$ extends to a homeomorphism of $\mathfrak{M}\left(H^{\infty}(\Delta)\right)$ onto $\overline{P(\xi)}$, which maps $\Gamma$ onto $\Gamma_{0}$.

Observe that $\Gamma_{0}$ is contained in $\overline{P(\xi)} \backslash P(\xi)$. We denote by $\lambda_{\omega}$ the representing measure for $\omega$ in $\Gamma_{0}$. Then $\lambda_{\omega}$ is invariant on the flow $\left(\mathbf{M},\left\{T_{t}\right\}_{t \in \mathbf{R}}\right)$ by (i) of Lemma 4.5. For each $\phi$ in $C(\mathbf{M})$, we set

$$
\tau \phi(\omega)=\int_{\mathbf{M}} \phi(x) d \lambda_{\omega}(x), \quad \omega \in \Gamma_{0} .
$$

Then the map $\omega \rightarrow \tau \phi(\omega)$ is continuous on $\Gamma_{0}$. Indeed, by identifying each $\omega$ in $\Gamma_{0}$ with its unique representing measure $\lambda_{\omega}$ in $M(\mathbf{M}), \Gamma_{0}$ becomes a subset of $M(\mathbf{M})=C(\mathbf{M})^{*}$, the dual space of $C(\mathbf{M})$. The weak- $*$ closure of $\left\{\lambda_{\omega} ; \omega \in \Gamma_{0}\right\}$ consists of invariant probability measures which are multiplicative on $A(\mathbf{M})$. Since $\Gamma_{0}$ is closed in $\mathfrak{M}(A(\mathbf{M}))$ and since representing measures 
are unique, the weak-* closure of $\left\{\lambda_{\omega} ; \omega \in \Gamma_{0}\right\}$ has to be itself. Then the map $\lambda_{\omega} \rightarrow \omega$ is continuous from one compact space to another, so the continuity of $\omega \rightarrow \tau \phi(\omega)$ follows. We observe easily that $\tau$ is a positive linear operator of $C(\mathbf{M})$ into $C\left(\Gamma_{0}\right)$ with $\|\tau\|=1$.

Let $\nu$ be a positive measure in $M\left(\Gamma_{0}\right)$. We then define the linear functional $F$ on $C(\mathbf{M})$ by

$$
F(\phi)=\int_{\Gamma_{0}} \tau \phi(\omega) d \nu(\omega), \quad \phi \in C(\mathbf{M}) .
$$

We see that $|F(\phi)| \leq\|\nu\| \cdot\|\phi\|_{\infty}$ and $F\left(T_{t} \phi\right)=F(\phi)$, where $T_{t} \phi(x)=\phi(x+t)$. We also see that $F$ is positive and $F(1)=\nu\left(\Gamma_{0}\right)=\|\nu\|$. Then the Riesz representation theorem assures the existence of a positive invariant measure $\mu$ in $M(\mathbf{M})$ such that

$$
F(\phi)=\int_{\mathbf{M}} \phi(x) d \mu(x), \quad \phi \in C(\mathbf{M}) .
$$

It is clear that $\mu$ is uniquely determined and $\|\mu\|=\|\nu\|$. However, $\nu$ may not be determined uniquely by $\mu$, which causes a difficulty.

We next extend $\tau$ to a positive linear operator of $L^{1}(\mu)$ to $L^{1}(\nu)$ with $\|\tau\|=1$. Let $\phi$ be a function in $C_{\mathbf{R}}(\mathbf{M})$. We write $\phi=\phi_{1}-\phi_{2}$, where $\phi_{1}$ and $\phi_{2}$ are respectively the positive and negative parts of $\phi$. Since $\left|\tau \phi_{1}-\tau \phi_{2}\right| \leq$ $\tau \phi_{1}+\tau \phi_{2}$ and $|\phi|=\phi_{1}+\phi_{2}$, we have that $\|\tau \phi\|_{1} \leq\|\phi\|_{1}$. From this fact, the assertion follows easily. We denote also by $\tau$ this extended operator of $L^{1}(\mu)$ to $L^{1}(\nu)$.

Let $\tau^{*}$ be the adjoint of the extended operator $\tau$. Then $\tau^{*}$ is a positive linear operator of $L^{\infty}(\nu)$ to $L^{\infty}(\mu)$, and its range consists of invariant functions. When $\phi$ is in $L^{1}(\mu)$, we put

$$
\left\{\begin{array}{l}
m_{T} \phi(x)=\frac{1}{2 T} \int_{-T}^{T} \phi(x+t) d t, \text { and } \\
m \phi(x)=\lim _{T \rightarrow \infty} m_{T} \phi(x) .
\end{array}\right.
$$

Then the mean ergodic theorem shows that $m \phi$ lies in $L^{1}(\mu)$ and $\tau(m \phi)=\tau \phi$. We see also that $m_{T} \phi$ lies in $C(\mathbf{M})$ if $\phi$ is in $C(\mathbf{M})$.

Let $b(z)$ be a Blaschke product on $\Delta$. By Lemma 4.6, we may choose a decreasing sequence $\left\{r_{n}\right\}$ converging to 1 and a sequence $\left\{B_{n}\right\}$ of Blaschke products on $\mathbf{R}_{+}^{2}$ such that

$$
r_{n} B_{n} \circ L_{0}(\eta)=b(\eta), \quad \eta \in \mathfrak{M}\left(H^{\infty}(\Delta)\right),
$$

where $L_{0}$ is identified with its extension to $\mathfrak{M}\left(H^{\infty}(\Delta)\right)$ by Lemma 4.8. This yields that $\tau\left(r_{n} B_{n}\right)(\omega)=b \circ L_{0}^{-1}(\omega)$ on $\Gamma_{0}$. Since $b \circ L_{0}^{-1}$ lies in $L^{\infty}(\nu)$, $\tau^{*}\left(b \circ L_{0}^{-1}\right)(x)$ is an invariant function in $L^{\infty}(\mu)$. We claim that

$$
\tau \circ \tau^{*}\left(b \circ L_{0}^{-1}\right)(\omega)=b \circ L_{0}^{-1}(\omega), \quad \omega \in \Gamma_{0} .
$$

Indeed, if we set $\phi_{n}=m\left(r_{n} B_{n}\right)$ by (4.30), then $\phi_{n}$ is an invariant function in $L^{\infty}(\mu)$ with $\left\|\phi_{n}\right\|_{\infty} \leq r_{n}$ and satisfies that $\tau \phi_{n}=b \circ L_{0}^{-1}$ on $\Gamma_{0}$. So we have that

$$
\|\nu\|=\int_{\Gamma_{0}}\left|b \circ L_{0}^{-1}\right|^{2} d \nu=\int_{\Gamma_{0}}\left(\tau \bar{\phi}_{n}\right)\left(b \circ L_{0}^{-1}\right) d \nu=\int_{\mathbf{M}} \bar{\phi}_{n} \tau^{*}\left(b \circ L_{0}^{-1}\right) d \mu .
$$


Since $\left\|\tau^{*}\left(b \circ L_{0}^{-1}\right)\right\|_{\infty} \leq 1$ and $\|\mu\|=\|\nu\|$, this shows that

$$
\begin{aligned}
\left\|\phi_{n}-\tau^{*}\left(b \circ L_{0}^{-1}\right)\right\|_{2}^{2} & =\left\|\phi_{n}\right\|_{2}^{2}-2 \operatorname{Re} \int_{\mathbf{M}} \bar{\phi}_{n} \tau^{*}\left(b \circ L_{0}^{-1}\right) d \mu+\left\|\tau^{*}\left(b \circ L_{0}^{-1}\right)\right\|_{2}^{2} \\
& \leq r_{n}^{2}\|\mu\|-2\|\mu\|+\|\mu\|=\left(r_{n}^{2}-1\right)\|\mu\| .
\end{aligned}
$$

Since $\left\|\phi_{n}-\tau^{*}\left(b \circ L_{0}^{-1}\right)\right\|_{1} \leq\left(r_{n}^{2}-1\right)^{1 / 2}\|\mu\|$ and $\tau \phi_{n}=b \circ L_{0}^{-1}$, we see that $\tau \circ \tau^{*}\left(b \circ L_{0}^{-1}\right)=b \circ L_{0}^{-1}$. Thus we obtain the following:

Lemma 4.9. Let $L_{0}, \tau$, and $\tau^{*}$ be as in above. If $b_{1}$ and $b_{2}$ are Blaschke products on $\Delta$, then we have the equation

$$
\int_{\Gamma_{0}}\left(b_{1} \circ L_{0}^{-1}\right)\left(\bar{b}_{2} \circ L_{0}^{-1}\right) d \nu=\int_{\mathbf{M}} \tau^{*}\left(b_{1} \circ L_{0}^{-1}\right) \tau^{*}\left(\bar{b}_{2} \circ L_{0}^{-1}\right) d \mu .
$$

Setting $b_{1}=b_{2}=b$ in Lemma 4.9 , we see that $\tau^{*}\left(b \circ L_{0}^{-1}\right)$ is a unimodular function.

Under these observations, we may show that $A(\mathbf{M})$ is not a Dirichlet algebra on $\mathbf{M}$. The following proof is due to the referee, which improves our former one so much.

Proof of Theorem 4.1. It suffices to show that there is a nonzero real measure in $M(\mathbf{M})$ which is orthogonal to $A(\mathbf{M})$. It is well known that $\widehat{H}^{\infty}(\Delta)$ is not a Dirichlet algebra on its Šilov boundary $\Gamma$ (see, for example, [8, Chapter 10]). By Lemma 4.7, we see that $\left.A\right|_{\overline{P(\xi)}}$ is not a Dirichlet algebra on $\Gamma_{0}$. Consequently, we may find a nonzero real measure $\nu$ in $M\left(\Gamma_{0}\right)$ which is orthogonal to $\left.A\right|_{\overline{P(\xi)}}$. By the Jordan decomposition theorem, $\nu$ is represented as $\nu=\nu_{1}-\nu_{2}$, where $\nu_{1}$ and $\nu_{2}$ are positive and negative variations of $\nu$. We may assume that both $\nu_{1}$ and $\nu_{2}$ are probability measures on $\Gamma_{0}$. It follows from (4.29) that there are invariant probability measures $\mu_{i}, i=1,2$, such that

$$
\int_{\Gamma_{0}} \tau \phi d \nu_{i}=\int_{\mathbf{M}} \phi d \mu_{i}, \quad \phi \in C(\mathbf{M}) .
$$

Let $\tau_{i}$ denote the extension of $\tau$ to $L^{1}\left(\mu_{i}\right)$, as described in above. The adjoint of $\tau_{i}$ is similarly denoted by $\tau_{i}^{*}$. Let $0<\varepsilon<\frac{1}{3}$. Since $\nu_{1}$ and $\nu_{2}$ are mutually singular, we may choose a unimodular continuous function $\theta$ on $\Gamma_{0}$ such that

$$
\left|\int_{\Gamma_{0}} \theta d\left(\nu_{1}-\nu_{2}\right)\right| \geq\left\|\nu_{1}\right\|+\left\|\nu_{2}\right\|-\varepsilon=2-\varepsilon .
$$

Let $L^{\infty}(\mathbf{T})$ be the space of bounded (Lebesgue) measurable functions on the unit circle $\mathbf{T}$. Since $\Gamma$ is the maximal ideal space of $L^{\infty}(\mathbf{T}), \theta \circ L_{0}$ is regarded as a unimodular function in $L^{\infty}(\mathbf{T})$. Then the Douglas-Rudin theorem and Frostman's theorem show that there are Blaschke products $b_{1}$ and $b_{2}$ on $\Delta$ such that $\left\|\theta \circ L_{0}-b_{1} \bar{b}_{2}\right\|_{\infty}<\frac{1}{2} \varepsilon$ (see [6, Chapter 5, Theorem 2.1]). This implies that

$$
\left|\int_{\Gamma_{0}}\left(b_{1} \circ L_{0}^{-1}\right)\left(\bar{b}_{2} \circ L_{0}^{-1}\right) d\left(\nu_{1}-\nu_{2}\right)\right| \geq 2-2 \varepsilon .
$$

By Lemma 4.9, we see that

$$
\left|\int_{\mathbf{M}} \tau_{1}^{*}\left(b_{1} \circ L_{0}^{-1}\right) \tau_{1}^{*}\left(\bar{b}_{2} \circ L_{0}^{-1}\right) d \mu_{1}-\int_{\mathbf{M}} \tau_{2}^{*}\left(b_{1} \circ L_{0}^{-1}\right) \tau_{2}^{*}\left(\bar{b}_{2} \circ L_{0}^{-1}\right) d \mu_{2}\right| \geq 2-2 \varepsilon .
$$


There are two sequences of Blaschke products $\left\{B_{n}^{(1)}\right\}$ and $\left\{B_{n}^{(2)}\right\}$ on $\mathbf{R}_{+}^{2}$ and two decreasing sequences $\left\{r_{n}^{(1)}\right\}$ and $\left\{r_{n}^{(2)}\right\}$ converging to 1 such that each $B_{n}^{(j)}$, $j=1,2$, extends to be continuous on $\mathbf{X}$ and $r_{n}^{(j)} B_{n}^{(j)} \circ L_{0}=b_{j}$ on $\mathfrak{M}\left(H^{\infty}(\Delta)\right)$. Let $m_{T}$ and $m_{i}$ be the operators by (4.30) with respect to $L^{1}\left(\mu_{i}\right)$. Recall that, for $\phi$ in $C(\mathbf{M}), m_{T}(\phi)$ lies in $C(\mathbf{M})$ and $\left\|m_{T}(\phi)-m_{i}(\phi)\right\|_{2}$ converges to 0 as $T \rightarrow \infty$. On the other hand, from the paragraph preceding Lemma 4.9, we see that

$$
\lim _{n \rightarrow \infty} \int_{\mathbf{M}}\left|m_{i}\left(r_{n}^{(j)} B_{n}^{(j)}\right)-\tau_{i}^{*}\left(b_{j} \circ L_{0}^{-1}\right)\right|^{2} d \mu_{i}=0
$$

for $i, j=1,2$. Hence there exists a $k$ such that

$$
\left|\int_{\mathbf{M}} m_{1}\left(r_{k}^{(1)} B_{k}^{(1)}\right) m_{1}\left(r_{k}^{(2)} \bar{B}_{k}^{(2)}\right) d \mu_{1}-\int_{\mathbf{M}} m_{2}\left(r_{k}^{(1)} B_{k}^{(1)}\right) m_{2}\left(r_{k}^{(2)} \bar{B}_{k}^{(2)}\right) d \mu_{2}\right| \geq 2-3 \varepsilon .
$$

Therefore there is a $T>0$ such that

$$
\left|\int_{M} m_{T}\left(r_{k}^{(1)} B_{k}^{(1)}\right) m_{T}\left(r_{k}^{(2)} \bar{B}_{k}^{(2)}\right) d\left(\mu_{1}-\mu_{2}\right)\right| \geq 2-4 \varepsilon>\frac{2}{3} .
$$

Since $m_{T}\left(r_{k}^{(j)} B_{k}^{(j)}\right)$ is in $C(\mathbf{M})$, this shows that $\mu_{1} \neq \mu_{2}$. Since $\nu=\nu_{1}-\nu_{2}$ is orthogonal to $\left.A\right|_{\overline{P(\xi)}}$, we obtain a nonzero real measure $\mu=\mu_{1}-\mu_{2}$ in $M(\mathbf{M})$ which is orthogonal to $A(\mathbf{M})$. This completes the proof.

\section{REMARKS}

Let $\left(\mathbf{M},\left\{T_{t}\right\}_{t \in \mathbf{R}}\right), A(\mathbf{M})$, and $P(\xi)$ be as in $\S 4$, and let $\mu$ be the representing measure for $\xi$. Then $\mu$ is an invariant probability measure in $M(\mathbf{M})$ which is not ergodic. We denote by $H^{\infty}(\mu)$ the weak-* closure of $A(\mathbf{M})$ in $L^{\infty}(\mu)$. Then $H^{\infty}(\mu)$ is a weak-* Dirichlet algebra in $L^{\infty}(\mu)$, since $A(\mathbf{M})$ is a logmodular algebra.

(i) Recall that, since $\left(\mathbf{M},\left\{T_{t}\right\}_{t \in \mathbf{R}}\right)$ is minimal, $A(\mathbf{M})$ is a maximal subalgebra of $C(\mathbf{M})$. By contrast to this fact, it follows from [11, Corollary 3.1] that $H^{\infty}(\mu)$ is not a weak-* maximal algebra in $L^{\infty}(\mu)$.

(ii) For each $\phi$ in $L^{1}(\mu), m \phi$ denotes the invariant function by (4.30). We now set

$$
\mathscr{H}=\{m \phi ; \phi \in A(\mathbf{M})\} .
$$

We then see that $\mathscr{H}$ is isometrically isomorphic to $H^{\infty}(\Delta)$. Of course, $m \phi$ cannot be continuous on $\mathbf{M}$ except for constants.

(iii) Let $\left(\Omega,\left\{U_{t}\right\}_{t \in \mathbf{R}}\right)$ be a minimal flow. Recall that if $\left(\Omega,\left\{U_{t}\right\}_{t \in \mathbf{R}}\right)$ is strictly ergodic, then $A(\Omega)$ is a Dirichlet algebra on $\Omega$. Then it is natural to ask whether strict ergodicity is necessary when $A(\Omega)$ is a Dirichlet algebra. We may, however, construct a minimal flow $\left(\mathbf{Y},\left\{V_{t}\right\}_{t \in \mathbf{R}}\right)$ not being strictly ergodic, on which $A(\mathbf{Y})$ is a Dirichlet algebra. Indeed, let $B_{1}(z)$ be the Blaschke product in (4.16) with $m=1$. Since $B_{1}(z)$ extends to be continuous on $\mathbf{X}$, it determines a unimodular function $B_{1}(x)$ in $A(\mathbf{M})$. Let $\mathfrak{B}$ be the closed subalgebra of $C(\mathbf{M})$ generated by $\left\{T_{t} B_{1}, \overline{T_{s} B_{1}} ; t, s \in \mathbf{R}\right\}$. Taking a suitable factor of $\left(\mathbf{M},\left\{T_{t}\right\}_{t \in \mathbf{R}}\right)$, we have a minimal flow $\left(\mathbf{Y},\left\{V_{t}\right\}_{t \in \mathbf{R}}\right)$ such that $\mathfrak{B}$ is isometricaly isomorphic to $C(\mathbf{Y})$. We may consider that $A(\mathbf{Y})$ contains the uniform algebra generated by $\left\{T_{t} B_{1} ; t \in \mathbf{R}\right\}$ and all the constant functions. Then it is not difficult to show that $\left(\mathbf{Y},\left\{V_{t}\right\}_{t \in \mathbf{R}}\right)$ is the desired one. We mention that since $C(\mathbf{Y})$ is separable, $\mathbf{Y}$ is metrizable. 
Incidentally, the referee has pointed out that there can be a minimal flow $(\Omega$, $\left.\left\{U_{t}\right\}_{t \in \mathbf{R}}\right)$ where $\Omega$ is metrizable, and yet $A(\Omega)$ is not a Dirichlet algebra. In this situation, Choquet's theorem works well. So we would expect some information about the invariant measures representing homomorphisms of $A(\Omega)$.

Note added in proof. One application of the flow $\left(\mathbf{X},\left\{\mathbf{S}_{t}\right\}_{t \in \mathbf{R}}\right)$ should be noted: Let $\mu$ be an invariant, ergodic, probability measure in $M(\mathbf{X})$, and let $H^{2}(\mu)$ and $H_{0}^{2}(\mu)$ be as ian the paragraph preceding Proposition 3.2. Then the invariant subspace $H_{0}^{2}(\mu)$ is generated by one of its elements. This answers an old question in the setting of weak- ${ }^{*}$ Dirichlet algebras (see [7, Chapter 5, §4]). For the proof, see our subsequent note Single generator problem.

\section{REFERENCES}

1. N. Dunford and J. Schwartz, Linear operators. Part I, Wiley Interscience, New York, 1958.

2. F. Forelli, Analytic and quasi-invariant measures, Acta Math. 118 (1967), 33-59.

3. __ Fourier theory and flows, Actes, Congrès Internat. Math. 2 (1970), 475-476.

4. __ A maximal algebra, Math. Scand. 30 (1970), 152-158.

5. T. Gamelin, Uniform algebras, Prentice-Hall, Englewood Cliffs, N.J., 1969.

6. J. Garnett, Bounded analytic functions, Academic Press, New York, 1981.

7. H. Helson, Analyticity on compact abelian groups, Algebras in Analysis, Academic Press, London, 1975, pp. 1-62.

8. K. Hoffman, Banach spaces of analytic functions, Prentice-Hall, Englewood Cliffs, N.J., 1962.

9. $ـ$ Bounded analytic functions and Gleason parts, Ann. of Math. (2) 86 (1967), 74-111.

10. D. Marshall, Blaschke products generate $H^{\infty}$, Bull. Amer. Math. Soc. 82 (1967), 494-496.

11. P. Muhly, Function algebras and flows, Acta Sci. Math. (Szeged) 35 (1973), 111-121.

12. __ Function algebras and flows. II, Ark. Mat. 11 (1973), 203-213.

13. _ Function algebras and flows. IV, Trans. Amer. Math. Soc. 203 (1975), 55-66.

14. __ Isometries of ergodic Hardy spaces, Israel J. Math. 36 (1980), 50-74.

15. J. Tanaka, Corona problem and flows, J. Funct. Anal. 102 (1991), 360-378.

16. J. Xia, Conditional expectations and the corona problem of ergodic Hardy spaces, J. Funct. Anal. 64 (1985), 251-274.

Department of Mathematics, Tsuru University, Tsuru City, Yamanashi 402, Japan 